Communications in Statistics - Simulation and Computation

\title{
Optimal Design of Acceptance Sampling Plans by Variables for Nonconforming Proportions When the Standard Deviation Is Unknown
}

\section{Belmiro P.M. Duarte \& Pedro M. Saraiva}

To cite this article: Belmiro P.M. Duarte \& Pedro M. Saraiva (2013) Optimal Design of Acceptance Sampling Plans by Variables for Nonconforming Proportions When the Standard Deviation Is Unknown, Communications in Statistics - Simulation and Computation, 42:6, 1318-1342, DOI: 10.1080/03610918.2012.665548

To link to this article: https://doi.org/10.1080/03610918.2012.665548

曲 Published online: 10 Jan 2013.

Submit your article to this journal $2 \pi$

Llll Article views: 108

Citing articles: 6 View citing articles $₫$ 


\title{
Optimal Design of Acceptance Sampling Plans by Variables for Nonconforming Proportions When the Standard Deviation Is Unknown
}

\author{
BELMIRO P.M. DUARTE ${ }^{1}$ AND PEDRO M. SARAIVA ${ }^{2}$ \\ ${ }^{1}$ Department of Chemical and Biological Engineering, Instituto Superior de \\ Engenharia de Coimbra (ISEC), Polytechnic Institute of Coimbra, Coimbra, \\ Portugal \\ ${ }^{2}$ GEPSI - PSE Group, Chemical Process Engineering and Forest Products \\ Research Centre (CIEPQPF), Department of Chemical Engineering, University \\ of Coimbra Pólo II, Coimbra, Portugal
}

This article presents an optimization-based approach for the design of acceptance sampling plans by variables for controlling nonconforming proportions when the standard deviation is unknown. The variables are described by rigorous noncentral Student's $\mathrm{t}$-distributions. Single and double acceptance sampling (AS) plans are addressed. The optimal design results from minimizing the average sampling number (ASN), subject to conditions holding at producer's and consumer's required quality levels. The problem is then solved employing a nonlinear programming solver. The results obtained are in close agreement with previous sampling plans found in the literature, outperforming them regarding the feasibility.

Keywords Acceptance sampling plans by variables; Nonconforming proportions; Nonlinear programming; Unknown standard deviation.

Mathematics Subject Classification 62P30, 90C30, 90C90.

\section{Introduction}

Acceptance sampling (AS) is an effective quality control tool that has emerged in the past decades as one of the keystones of supplier-customer relationships (Evans, 2005). AS is essentially an auditing tool rather than a process development approach (Mitra, 1998), since it deals with the decision of accepting/rejecting a lot of items based on a sample. AS procedures can be applied to lots of items when testing reveals nonconformance or nonconformities regarding product functional attributes. It can also be applied to variables characterizing lots, revealing how far product quality is from specifications. Both AS applications have the basic purpose of classifying a lot as accepted or rejected, given the quality level required. The advantages of AS plans by variables are related with lower sample sizes required to reach a given operating characteristic (OC) curve discrimination

Received November 24, 2009; Accepted February 6, 2012

Address correspondence to Belmiro P. M. Duarte, Department of Chemical and Biological Engineering, Instituto Superior de Engenharia de Coimbra (ISEC), Polytechnic Institute of Coimbra, Rua Pedro Nunes, 3030-199, Coimbra, Portugal; E-mail: bduarte@ @isec.pt 
level compared with equivalent procedures for attributes (Schilling, 1982). This feature is particularly relevant when tests are destructive and the inspection cost is significant. However, AS plans by variables are often more difficult to implement, due to the lack of knowledge to set the basic assumptions that have to be met. The implementation requires different procedures to check lots regarding each of the variables controlled and each specification. Seidel (1997) analyzed the pros and cons, and proved that sampling by variables is optimal, and should be adopted whenever possible.

The design of AS plans by variables consists in the determination of the size of the samples to inspect and of the acceptance threshold to accept/reject a lot, called acceptance constant (Duncan, 1974). The initial algorithms employed were based on the concept of OC curve, using the risks of acceptance stipulated by the producer and the consumer to find feasible combinations of parameters. Such designs result from the solution of the equations modeling the acceptance probability in the controlled points on the OC curve, corresponding to producer's and consumer's risks (Bowker and Goode, 1952). Taking advantage of the growth of the calculation power in last decades, several algorithms were developed to handle the problem computationally (see the work of Sommers (1981)). Since the system of algebraic equations resulting from controlling the quality levels stipulated does not have a closed form for double sampling plans, most of the algorithms developed to this class of problems are based on enumeration techniques to seek feasible combinations of variables (Hilbert, 2005; Sommers, 1981). The design of AS plans by variables when the standard deviation of the process is known is perfectly established in several quality control references (Duarte and Saraiva, 2010; Feldmann and Krumbholz, 2002; Schilling, 1982). Those approaches assume that the statistic used for testing to reach the decision of accepting/rejecting the lot is normally distributed, and use the conditions at producer's and consumer's quality levels to determine the sample size and the AS constant. Enumerative algorithms allow to check the feasibility of the designs regarding both controlled points but do not guarantee tighter solutions. To handle the problem in a systematic way, employing mathematical programming approaches, Duarte and Saraiva (2010) proposed an optimization-based framework relying on robust optimization solvers. The design problem is convex, and this strategy allows to reach optimal plans with respect to a given criterion while simultaneously satisfying the constraints.

The design of AS plans with unknown standard deviation is an open topic in AS theory. Most of the strategies used to deal with this case assume that the statistic employed for testing follows an approximate normal distribution, instead of the noncentral Student's $t$-distribution, which indeed models the risk, to avoid the increase in the complexity of the calculation. The design of double sampling plans by variables with unknown standard deviation was addressed by Krumbholz and Rohr (2006), who developed exact representational forms for determining the OC curve.

In Section 2, we introduce the motivation for this work, particularly the findings of the infeasibility of AS plans available in the open literature for the case of $\sigma$ unknown, designed assuming that noncentral Student's $t$-distribution is approximated by normal approximations proposed by Wallis (1947). Hamaker (1979) improved the approximation proposed by Wallis (1947). However, to compare our results with those of Sommers (1981), the former was considered.

Our analysis revealed that there is room to improve the design of AS plans for processes with unknown standard deviation by using the rigorous representation of the noncentral Student's $t$-statistic to generate the OC curve. Its use, enclosed in mathematical programming-based approaches supported by robust optimization algorithms, guarantees feasible AS plans, thus producing designs that simultaneously and systematically address 
both conditions at the points on the OC curve. The exact representation of the noncentral Student's $t$-statistic for single sampling plans relies on a series expansion developed during the decades of 1960-70. The design of double sampling plans involving bivariate noncentral Student's $t$-distributions with different noncentrality parameters in both dimensions is handled by employing the OC curve developed by Krumbholz and Rohr (2006), combined with numerical integration techniques. The optimization-based approach presented in Duarte and Saraiva (2010) for determining double AS plans for $\sigma$ unknown by employing normal approximations is extended to consider exact noncentral Student's $t$-distribution. Similarly, the plans determined minimize the average sampling number (ASN), and the noncentral Student's $t$-cumulative distribution function (cdf) is computed via the algorithm of Lenth (1989), which stands in a series expansion of incomplete Beta functions.

The article is structured as following. Section 2 presents the basics of sampling theory for lot acceptance purposes. Section 3 presents the framework for designing single sampling plans by variables when $\sigma$ is unknown. Section 4 extends the formulation to deal with double sampling plans. Finally, Section 5 enumerates conclusions.

\section{Acceptance Sampling Theory Basics and Motivation}

To establish the nomenclature, we first introduce the conceptual basis of single AS plans by variables for controlling nonconforming proportions. We consider a system described by a quality characteristic of a large-size lot of a given product, represented by $X$, with $X \sim \mathcal{N}\left(\mu, \sigma^{2}\right)$, where $\mu$ is the average and $\sigma$ the standard deviation of $X$, with the value of $\sigma$ unknown, and thus approximated by the standard deviation of the sample, denoted as $s$. We also assume that there is a one-sided upper specification $U>0$, with the proportion of nonconforming product, $p$, given as

$$
1-p=\Phi\left(\frac{U-\mu}{\sigma}\right)
$$

where $\Phi(x)$ stands for the cdf of the normal distribution $\mathcal{N}(0,1)$ at $x$ :

$$
\Phi(x)=\frac{1}{\sqrt{2 \pi}} \int_{-\infty}^{x} \exp \left(-t^{2} / 2\right) \mathrm{d} t
$$

Here, we confine the strategy presented to AS plans for controlling quality variables with an upper specification. However, the extension to plans for variables with a lower specification is straightforward. From Eq. (1), one obtains

$$
z_{p}=\Phi^{-1}(1-p)=\frac{U-\mu}{\sigma}
$$

with $\Phi^{-1}(1-p)$ standing for the inverse of the cumulative standard normal distribution representing a proportion $1-p$. We call the acceptance probability required at the quality level $p$ as $P$. The producer's quality level is to be designated as $p_{1}$, and the consumer's quality level as $p_{2}$, with $p_{1}<p_{2}$. Typical AS plans are designed to meet two simultaneous conditions, known as the OC curve two-point conditions:

1. The consumer accepts lots of quality level $p_{1}$ no less than $P_{1} \%$ of the times, with $p_{1}$ called the acceptable quality level (AQL) and $P_{1}$ represented by $1-\alpha$. 
2. The producer accepts lots of quality level $p_{2}$ no more than $P_{2} \%$ of the times, with $p_{2}$ called the limit quality level (LQL) and $P_{2}$ represented by $\beta$.

In Section 3, we address the design of single sampling plans with a single specification, compactly represented as $\mathcal{S}_{1}\left(n_{s 1} \quad k_{a}\right)$, where $n_{s 1} \in \mathbb{N}$ stands for the sample size and $k_{a} \in \mathbb{R}$ is the acceptance constant.

The mechanics of the single sampling plan with an upper specification and $\sigma$ unknown is described as follows:

1. Sample $n_{s 1}$ items of a lot and determine the quality characteristic of each one, $X_{1}, \ldots, X_{n_{s 1}}$.

2. Determine the average, $\bar{X}$, and the standard deviation, $s$, of the sample, where

$$
\bar{X}=\frac{1}{n_{s 1}} \sum_{i=1}^{n_{s 1}} X_{i} ; \quad s=\sqrt{\frac{\sum_{i=1}^{n_{s 1}}\left(X_{i}-\bar{X}\right)^{2}}{n_{s 1}-1}} .
$$

3. Determine $t_{1}=(U-\bar{X}) / s$.

4. If $t_{1} \geq k_{a}$, accept the lot; otherwise, reject it.

The OC curve represents the acceptance probability of a lot, denoted as $L(p)$, versus nonconforming proportion of the quality characteristic in the lot, denoted as $p$. Considering the mechanics of single AS plans presented above, $L(p)=P_{a}\left(T_{1} \geq \sqrt{n_{\sigma 1}} k_{a} \mid p\right)$, with $P_{a}$ standing for the acceptance probability, given a sampling acceptance constant, $k_{a}$. The OC curve for processes with $\sigma$ known is defined by Eq. (5), and is represented in Figure 1:

$$
L^{1}(p)=\Phi\left[\sqrt{n_{\sigma 1}}\left(\Phi^{-1}(1-p)-k_{a}\right)\right]
$$

where $n_{\sigma 1}$ is the sample size for plans with $\sigma$ known.

Figure 1 also serves to graphically identify the quality levels and corresponding acceptance probabilities used for designing sampling plans based on the OC curve two-point conditions paradigm, with the points denoted by $\left(p_{1}, P_{1}\right)$ and $\left(p_{2}, P_{2}\right)$, respectively.

For the case of $\sigma$ unknown, the following is observed:

$$
\frac{\sqrt{n_{s 1}}}{\sigma}(U-\bar{X}) \sim \mathcal{N}\left(\sqrt{n_{s 1}} \Phi^{-1}(1-p), 1\right),
$$

thus leading to

$$
\frac{\sqrt{n_{s 1}}}{\sigma}(U-\bar{X}) \stackrel{d}{=} \mathcal{N}(0,1)+\sqrt{n_{s 1}} \Phi^{-1}(1-p) .
$$

Since $\left(n_{s 1}-1\right) s^{2} / \sigma^{2}$ follows an independent $\chi^{2}$-distribution with $n_{s 1}-1$ degrees of freedom, the statistic $\sqrt{n_{s} 1}(U-\bar{X}) / s$ is described by noncentral Student's $t$-distribution with $n_{s 1}-1$ degrees of freedom and mean (also known as noncentrality parameter) equal to $\sqrt{n_{s 1}} \Phi^{-1}(1-p)$. Therefore, the OC curve for this class of plans becomes

$$
\begin{aligned}
L^{2}(p) & =\int_{\sqrt{n_{s 1}} k_{a}}^{+\infty} \psi\left[T_{1} \mid n_{s 1}-1, \sqrt{n_{s 1}} \Phi^{-1}(1-p)\right] \mathrm{d} T_{1} \\
& =1-\int_{-\infty}^{\sqrt{n_{s 1}} k_{a}} \psi\left[T_{1} \mid n_{s 1}-1, \sqrt{n_{s 1}} \Phi^{-1}(1-p)\right] \mathrm{d} T_{1} \\
& =\Psi\left[T_{1} \mid n_{s 1}-1, \sqrt{n_{s 1}} \Phi^{-1}(1-p)\right],
\end{aligned}
$$




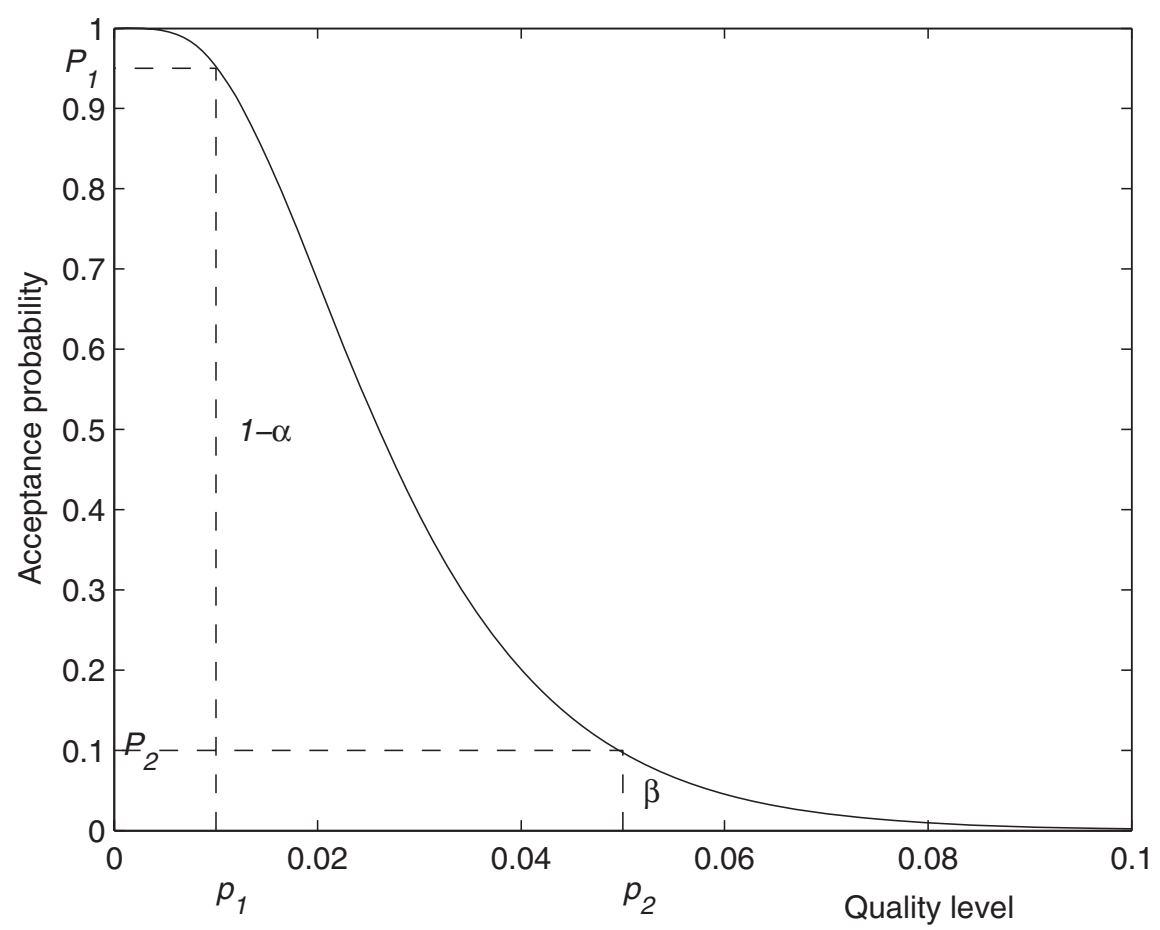

Figure 1. OC curve for single sampling plan $\mathcal{S}_{1}\left(\begin{array}{ll}19 & 1.943\end{array}\right)$.

where $\psi\left[T_{1} \mid n_{s 1}-1, \sqrt{n_{s 1}} \Phi^{-1}(1-p)\right]$ is the noncentral Student's $t$-probability density function (pdf) with $n_{s 1}-1$ degrees of freedom and mean $\sqrt{n_{s 1}} \Phi^{-1}(1-p)$ at $T_{1}$, and $\Psi\left[T_{1} \mid n_{s 1}-1, \sqrt{n_{s 1}} \Phi^{-1}(1-p)\right]$ is the corresponding cdf. Due to the complexity in determining noncentral Student's $t$-statistic cdf, several authors used an approximation based on the normal distribution $\mathcal{N}\left(\mu+k_{a} \sigma, \sigma^{2} \times\left(1 / n_{s 1}+\right.\right.$ $\left.\left.k_{a}^{2} /\left(2 n_{s 1}-1\right)\right)\right)$ proposed by Wallis (1947). The representational form of the OC curve becomes

$$
L^{3}(p)=\Phi\left\{\sqrt{n_{s 1}}\left[\Phi^{-1}(1-p)-k_{a}\right] \mid \sqrt{n_{s 1}} k_{a}, n_{s 1}\left[1 / n_{s 1}+k_{a}^{2} /\left(2 n_{s 1}-1\right)\right]\right\} .
$$

Here, $\Phi(x \mid a, b)$ stands for the cdf of the normal distribution with average $a$ and standard deviation $b$ at point $x$. A common strategy adopted to compute the OC curve represented by $L^{3}(p)$ (Schilling, 1982; Sommers, 1981) consists in using the form derived for the case of $\sigma$ known (Eq. 5), with $n_{\sigma 1}$ related to $n_{s 1}$ by

$$
n_{\sigma 1}=\frac{n_{s 1}}{1+k_{a}^{2} n_{s 1} /\left(2 n_{s 1}-1\right)},
$$

which leads to

$$
L^{3}(p)=\Phi\left[\sqrt{\frac{n_{s 1}}{1+k_{a}^{2} n_{s 1} /\left(2 n_{s 1}-1\right)}}\left(\Phi^{-1}(1-p)-k_{a}\right)\right] .
$$


Here, Eq. (11) is also used for single AS plans with $\sigma$ unknown. The computation of the cdf for noncentral Student's $t$-statistic is performed using the algorithm of Lenth (1989), which, in its turn, is based on the expansion proposed by Guenther (1978).

Let us consider for the sake of simplicity that $\sqrt{n_{s 1}} k_{a} \geq 0$. Therefore, the following equality holds:

$$
\begin{aligned}
& \int_{-\infty}^{\sqrt{n_{s 1}} k_{a}} \psi\left[T_{1} \mid n_{s 1}-1, \sqrt{n_{s 1}} \Phi^{-1}(1-p)\right] \mathrm{d} T_{1} \\
& \quad=\int_{-\infty}^{0} \psi\left[T_{1} \mid n_{s 1}-1, \sqrt{n_{s 1}} \Phi^{-1}(1-p)\right] \mathrm{d} T_{1} \\
& \quad+\int_{0}^{\sqrt{n_{s 1}} k_{a}} \psi\left[T_{1} \mid n_{s 1}-1, \sqrt{n_{s 1}} \Phi^{-1}(1-p)\right] \mathrm{d} T_{1}
\end{aligned}
$$

and the cdf calculation includes two terms, with $\int_{-\infty}^{0} \psi\left[T_{1} \mid n_{s 1}-1, \sqrt{n_{s 1}} \Phi^{-1}(1-p)\right] \mathrm{d} T_{1}=$ $\Phi\left[-\sqrt{n_{s 1}} \Phi^{-1}(1-p)\right]:$

$$
\begin{aligned}
& \Psi\left[\sqrt{n_{s 1}} k_{a} \mid n_{s 1}-1, \sqrt{n_{s 1}} \Phi^{-1}(1-p)\right]=\Phi\left[-\sqrt{n_{s 1}} \Phi^{-1}(1-p)\right] \\
& +\int_{0}^{\sqrt{n_{s 1}} k_{a}} \psi\left[T_{1} \mid n_{s 1}-1, \sqrt{n_{s 1}} \Phi^{-1}(1-p)\right] \mathrm{d} T_{1} .
\end{aligned}
$$

The second term is then approximated by an infinite-series expansion:

$$
\begin{aligned}
& \int_{0}^{\sqrt{n_{s 1}} k_{a}} \psi\left[T_{1} \mid n_{s 1}-1, \sqrt{n_{s 1}} \Phi^{-1}(1-p)\right] \mathrm{d} T_{1} \\
&=\sum_{j=0}^{+\infty}\left\{r_{j} I_{x}\left[j+0.5,\left(n_{s 1}-1\right) / 2\right]+q_{j} I_{x}\left[j+1,\left(n_{s 1}-1\right) / 2\right]\right\} \\
& I_{x}(a, b)=\frac{\Gamma(a+b)}{\Gamma(a) \Gamma(b)} \int_{0}^{x} T_{1}^{a-1}\left(1-T_{1}\right)^{b-1} \mathrm{~d} T_{1}, \\
& r_{j}=\frac{1}{2} \frac{\exp \left(-\delta^{2} / 2\right)}{j !}\left(\frac{\delta}{2}\right)^{j} \\
& q_{j}=\frac{1}{2 \sqrt{2}} \frac{\exp \left(-\delta^{2} / 2\right)}{\Gamma(j+1.5)}\left(\frac{\delta}{2}\right)^{j} \\
& \delta=\sqrt{n_{s 1}} \Phi^{-1}(1-p) \\
& x=\frac{n_{s 1} k_{a}^{2}}{n_{s 1} k_{a}^{2}+n_{s 1}-1},
\end{aligned}
$$

where $I_{x}(a, b)$ is the incomplete Beta distribution with $(a, b)$ degrees of freedom at $x$ and $\Gamma(a)$ is the $\Gamma$ function at $a$. The algorithm presented by Lenth (1989) to evaluate the integral of Eq. (14) stands on the addition of terms to the summation until a predefined accuracy is achieved. Since in our approach, the integral evaluation is enclosed in an optimization problem, the number of terms to include in the expansion is fixed a priori. Therefore, we 


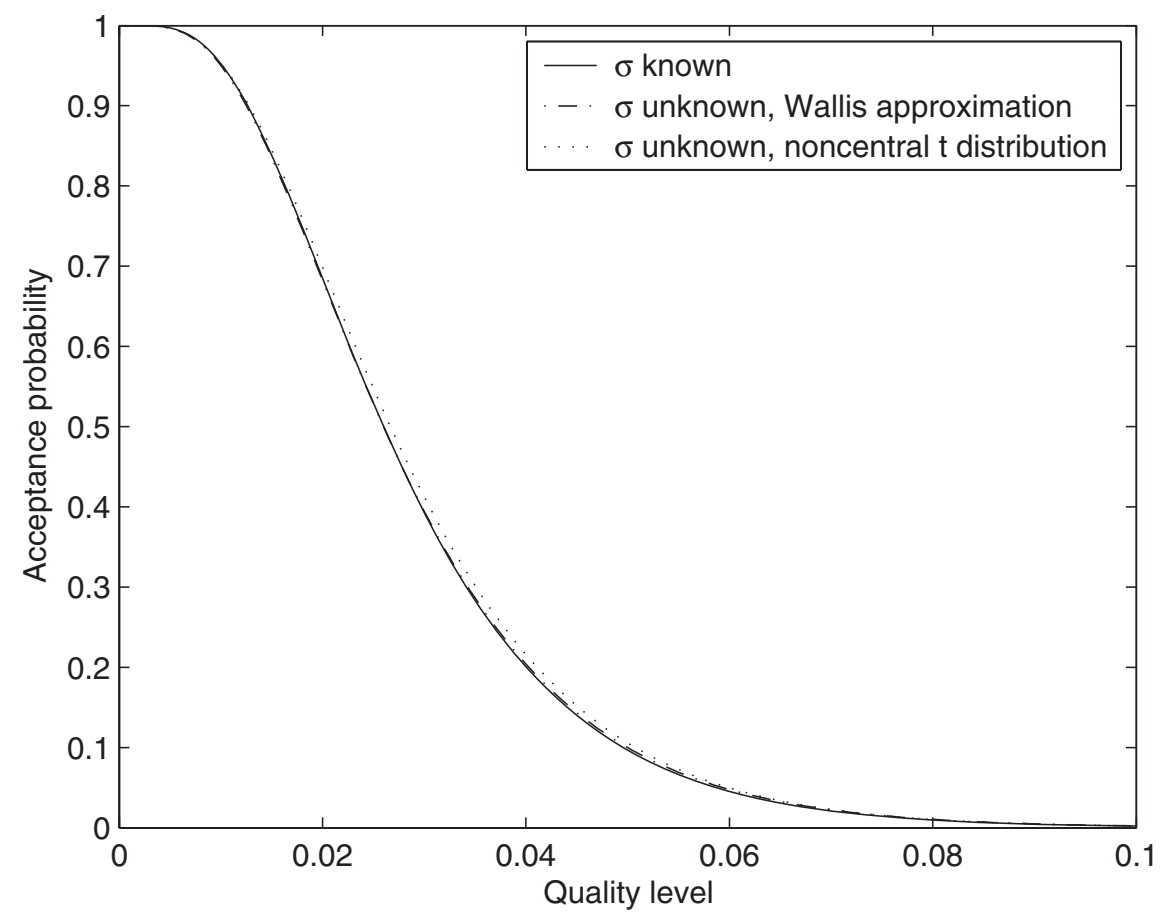

Figure 2. Comparison of OC curves for sampling plan $\mathcal{S}_{1}\left(\begin{array}{ll}19 & 1.943)\end{array}\right)$ determined for $\sigma$ known, sampling plan $\mathcal{S}_{1}\left(\begin{array}{ll}54 & 1.943\end{array}\right)$ determined for $\sigma$ unknown employing the normal approximation of Wallis (1947), and sampling plan $\mathcal{S}_{1}(54$ 1.943) determined for $\sigma$ unknown employing the exact Student's $t$-distribution.

have

$$
\begin{aligned}
& \int_{0}^{\sqrt{n_{s 1}} k_{a}} \psi\left[T_{1} \mid n_{s 1}-1, \sqrt{n_{s 1}} \Phi^{-1}(1-p)\right] \mathrm{d} T_{1} \\
& \quad \approx \sum_{j=0}^{M}\left\{r_{j} I_{x}\left[j+0.5,\left(n_{s 1}-1\right) / 2\right]+q_{j} I_{x}\left[j+1,\left(n_{s 1}-1\right) / 2\right]\right\},
\end{aligned}
$$

with the choice of $M$ discussed later in this section.

Figure 2 presents the OC curves for plans $\mathcal{S}_{1}\left(\begin{array}{ll}19 & 1.943\end{array}\right)$, designed for the case of $\sigma$ known, based on Eq. (5), the equivalent plan $\mathcal{S}_{1}(54$ 1.943) designed to address the case of $\sigma$ unknown employing the Wallis (1947) approximation (Eq. 11), and the plan $\mathcal{S}_{1}(541.943)$ based on the rigorous noncentral $t$-statistic represented by Eq. (8) and Eqs. (13-20). All AS plans were designed to meet $\mathrm{AQL}=0.01, \mathrm{LQL}=0.05, \alpha=0.05$, and $\beta=0.10$. The results obtained denote good agreement with those presented by Sommers (1981) based on the OC curve described by Eq. (11).

Figure 3 shows the difference between the OC curves generated for the cases of $\sigma$ unknown employing the normal approximation and noncentral Student's $t$-distribution. The difference is significant around the values of acceptance probability of 0.5 , being lower at the points controlled, corresponding to $(0.01,0.95)$ and $(0.05,0.10)$. A deeper analysis revealed that $L^{2}(0.01)=0.9528>0.95$, thus validating the constraint at the point $\left(p_{1}, 1-\alpha\right)$. 


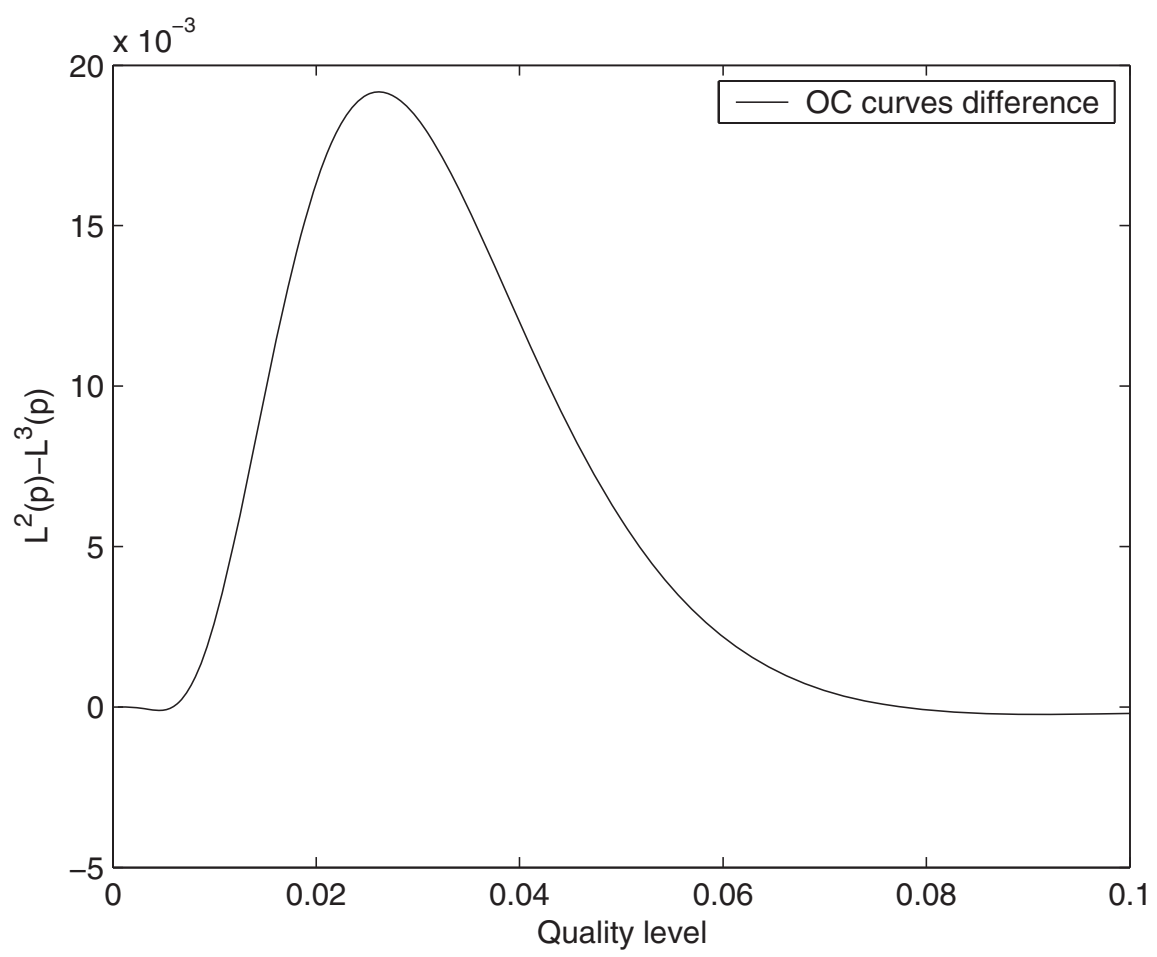

Figure 3. Difference between the OC curves for single sampling plan $\mathcal{S}_{1}\left(\begin{array}{ll}54 & 1.943\end{array}\right)$ obtained employing the noncentral $t$-distribution and the Wallis (1947) normal approximation.

However, $L^{2}(0.05)=0.1057>0.10$, thus violating the constraint at $\left(p_{2}, \beta\right)$. Therefore, we found that the AS plan based on the nonstandard normal distribution approximation is unfeasible. Afterward, our motivation will be to determine AS plans based on the noncentral Student's $t$-distribution to provide the research and industrial community with feasible optimal solutions.

The motivating case just analyzed allows us to predict that feasible AS plans derived based upon the OC curve $L^{2}(p)$ would lead to larger sample size and/or larger AS constant than those determined from $L^{3}(p)$.

The choice of the number of terms to include in the series expansion used to calculate noncentral Student's $t$-cdf is based on a heuristic rule. Lenth (1989) proved that 100 terms allow one to reach an accuracy of $10^{-6}$ for means ranging between -11.0 and 11.0. Here, the values of noncentrality parameter $\delta=\sqrt{n_{s 1}} \Phi^{-1}(1-p)$ may be larger than 11.0, thus requiring more than 100 terms. Figure 4 shows the values of $r_{j}, q_{j}, I_{x}\left[j+0.5,\left(n_{s 1}-1\right) / 2\right]$, $I_{x}\left[j+1,\left(n_{s 1}-1\right) / 2\right]$, and $\Psi\left[\sqrt{n_{s 1}} k_{a} \mid n_{s 1}-1, \sqrt{n_{s 1}} \Phi^{-1}(1-p)\right]$ for $M=150, n_{s 1}=54$, $\delta=14.278$, and $x=0.7937$. The terms that contribute more to the cdf are those arising from $j$ located in the neighborhood of $M / 2$, and the contribution is neglectful for terms of order higher than 130. Considering the results of Lenth (1989) and ours, one can adopt the following heuristic:

$$
M=\left\lceil n_{s 1} k_{a}^{2}\right\rceil,
$$

where $\lceil\bullet\rceil$ is the ceiling operator. 

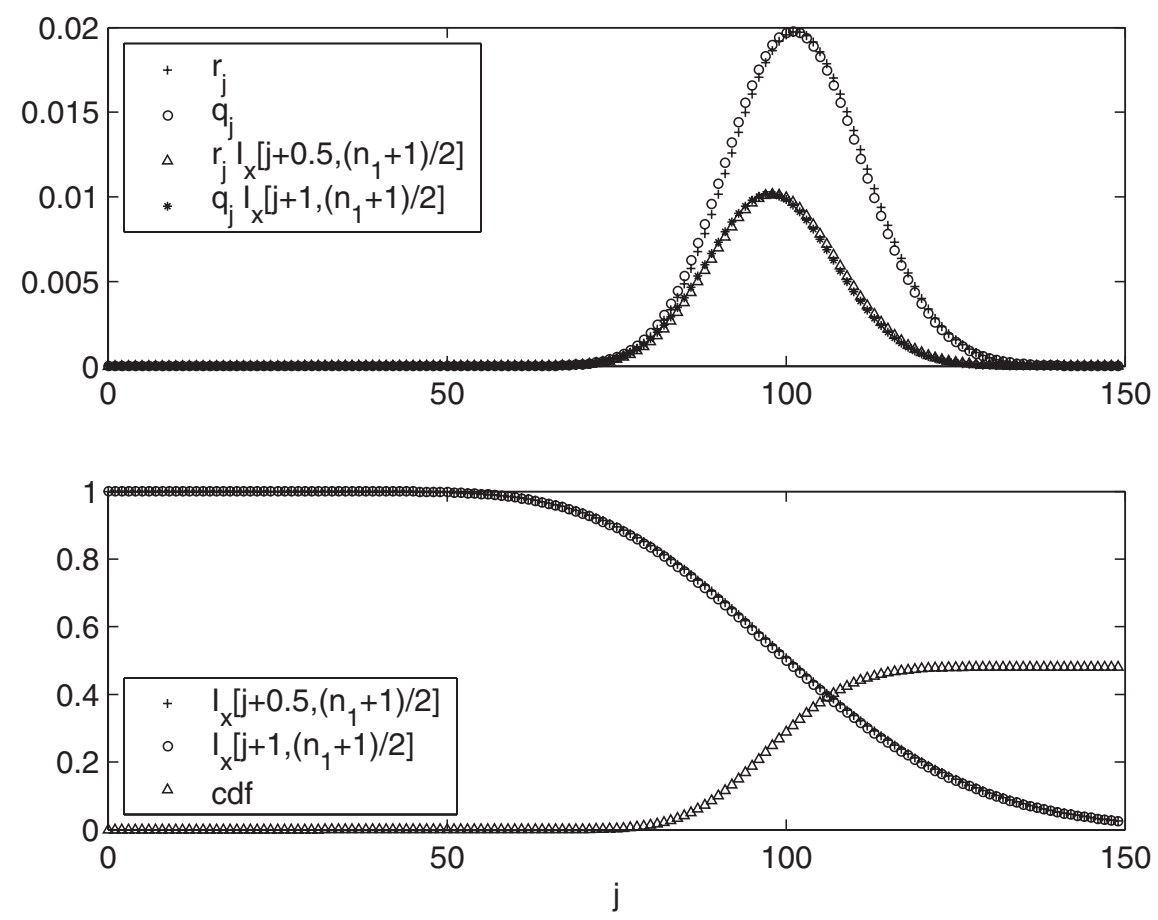

Figure 4. Terms involved in the expansion used to calculate noncentral Student's $t$-cdf.

\section{Single Acceptance Sampling Plans}

Considering the conceptual background introduced in Section 2, the design of single AS plans consists in the solution of an optimization problem aiming to minimize the sample size, subject to the constraints in the controlled points on the OC curve. The problem, denoted as $\mathcal{P}_{1 b}$, becomes

$$
\begin{array}{cl}
\min _{n_{s 1}, k_{a}} & n_{s 1} \\
\text { s.t. } & L^{2}\left(p_{1}\right) \geq 1-\alpha \\
& L^{2}\left(p_{2}\right) \leq \beta \\
& \text { Eqs. }(13-19) \\
& k_{a}>0, \quad n_{s 1} \geq 1,
\end{array}
$$

where $L^{2}(p)$ is determined with the series expansion methodology presented in Section 2 . The sample size is an integer variable, relaxed in problem $\mathcal{P}_{1 b}$ to avoid the need of using mixed integer-nonlinear solvers and providing analytical derivatives of the incomplete Beta function in case integer variables are used. The integer solution for sample size is determined from the relaxed solution of $\mathcal{P}_{1 b}$ by rounding it to the upper integer value. Since the AS design problem is convex, the solution so obtained does not affect the feasibility of the plan. A similar approach is followed in all problems addressed.

To lower the computation time required to obtain the solution of the problem $\mathcal{P}_{1 b}$, we first solve the equivalent problem based on the Wallis (1947) normal approximation, 
Table 1

Single sampling plans for $\alpha=0.05$ and $\beta=0.1$ ( $\sigma$ unknown) (values within parentheses correspond to plans derived by Sommers (1981))

\begin{tabular}{lcccccc}
\hline & & \multicolumn{2}{c}{ Wallis (1947) approximation } & & \multicolumn{2}{c}{ Noncentral $t$-distribution } \\
$p_{1}$ & $p_{2}$ & $n_{s}$ & $k_{a}$ & & $n_{s}$ & $k_{a}$ \\
\hline 0.02 & 0.030 & $835(835)$ & $1.957(1.96)$ & & 837 & 1.957 \\
0.02 & 0.035 & $417(417)$ & $1.918(1.92)$ & & 418 & 1.919 \\
0.02 & 0.040 & $259(259)$ & $1.883(1.88)$ & & 260 & 1.885 \\
0.02 & 0.045 & $182(182)$ & $1.852(1.85)$ & & 183 & 1.854 \\
0.02 & 0.050 & $137(137)$ & $1.824(1.82)$ & & 138 & 1.826 \\
0.02 & 0.060 & $89(89)$ & $1.773(1.77)$ & & 90 & 1.777 \\
0.02 & 0.070 & $65(65)$ & $1.729(1.73)$ & & 65 & 1.734 \\
0.02 & 0.080 & $50(50)$ & $1.689(1.69)$ & & 51 & 1.696 \\
0.02 & 0.090 & $41(41)$ & $1.653(1.65)$ & & 41 & 1.661 \\
0.02 & 0.100 & $34(34)$ & $1.620(1.62)$ & & 35 & 1.629 \\
0.02 & 0.110 & $29(29)$ & $1.589(1.59)$ & & 30 & 1.500 \\
0.02 & 0.120 & $25(25)$ & $1.560(1.56)$ & & 26 & 1.547 \\
0.02 & 0.130 & $22(22)$ & $1.533(1.53)$ & & 23 & 1.499 \\
0.02 & 0.150 & $18(18)$ & $1.482(1.48)$ & & 19 & 1.456 \\
0.02 & 0.170 & $15(15)$ & $1.436(1.44)$ & & 16 & 1.398 \\
0.02 & 0.200 & $12(12)$ & $1.372(1.37)$ & & 13 & \\
\hline
\end{tabular}

designated as problem $\mathcal{P}_{1 a}$ :

$$
\begin{array}{cl}
\min _{n_{s 1}, k_{a}} & n_{s 1} \\
\text { s.t. } & L^{3}\left(p_{1}\right) \geq 1-\alpha \\
& L^{3}\left(p_{2}\right) \leq \beta \\
& k_{a}>0, \quad n_{s 1} \geq 1 .
\end{array}
$$

The solution of $\mathcal{P}_{1 a}$ enables us to provide a feasible solution to handle the problem $\mathcal{P}_{1 b}$ and simultaneously set the number of terms used in the series expansion, with $M$ given by Eq. (21).

Both optimization problems fall into the category of nonlinear programming (NLP). Here, they are solved employing GAMS/CONOPT, which is a robust solver to handle large-scale NLP problems based on a generalized reduced-gradient algorithm (Drud, 1985), included in the GAMS optimization platform. The relative and absolute tolerances are set to $10^{-6}$ in all optimization problems addressed in the article.

Table 1 compares the sampling plans at $p_{1}=0.01, \alpha=0.05$, and $\beta=0.10$, for several values of LQL, available in the literature, with ours, obtained with noncentral Student's $t$-distribution. Our results require larger sample sizes (one additional item is required for all values of $p_{2}$ simulated) and slightly larger values of $k_{a}$. However, for single AS plans, the normal distribution is a good approximation for the statistic modeling the lot acceptance 


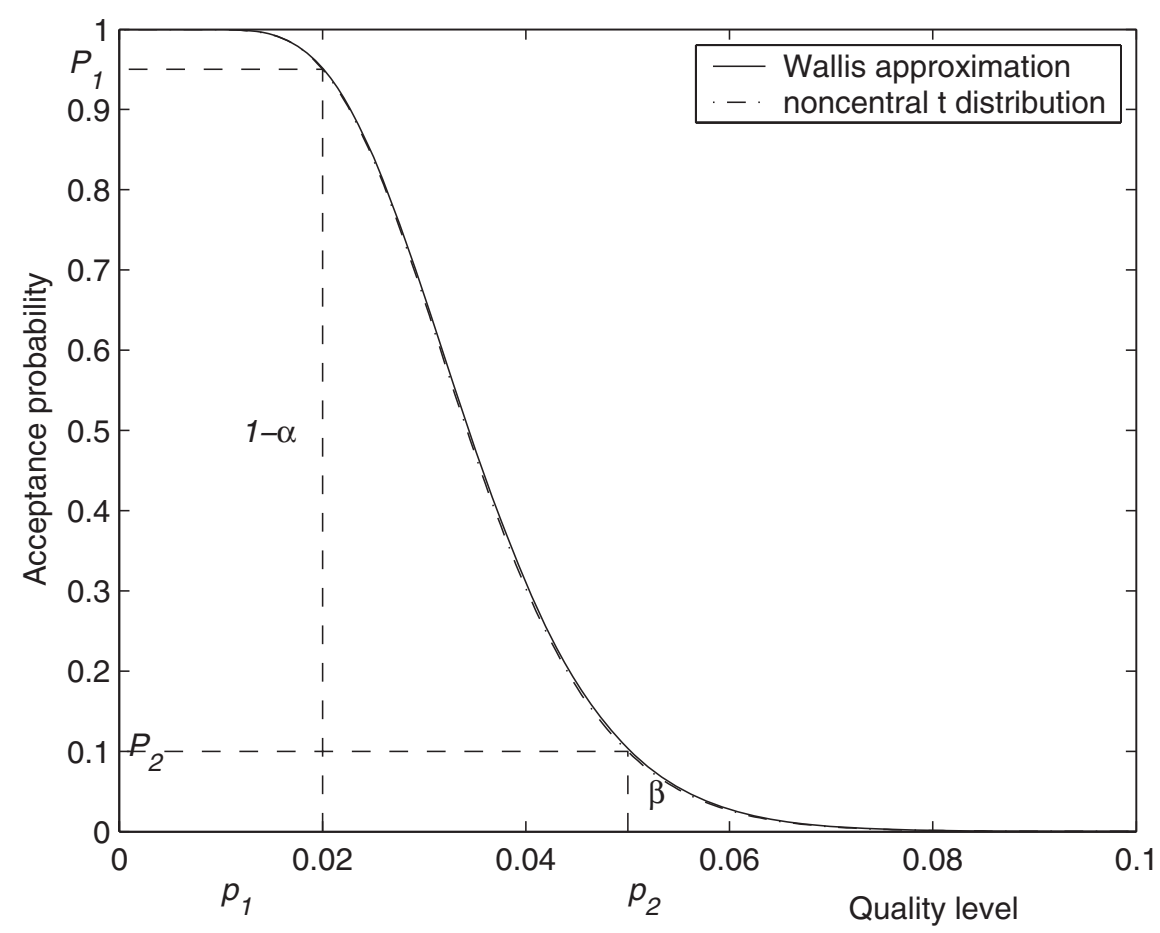

Figure 5. Comparison of OC curves for sampling plans $\mathcal{S}_{1}(137$ 1.824) based on the normal approximation of Wallis (1947) and sampling plan $\mathcal{S}_{1}(138$ 1.826) based on noncentral Student's $t$-distribution.

decision. This finding can be extended to the first stage of decision in double AS plans, once the numerical approach used to determine the acceptance probability is similar to ours.

The results presented here have the simple purpose of demonstrating the application of our strategy to single AS plans. Extensive tables can be easily generated by changing the parameters fixed. Figure 5 presents the comparison of OC curves for plans derived to meet $\mathrm{AQL}=0.02, \mathrm{LQL}=0.05, \alpha=0.05$, and $\beta=0.10$. The $\mathrm{OC}$ curves are almost coincident, but the plan based on normal distribution leads to $L^{2}(0.02)=0.9516$ and $L^{2}(0.05)=$ 0.1036 , thus violating the constraint at $p_{2}$. Both conditions hold for the plan based on noncentral Student's $t$-distribution with $L^{2}(0.02)=0.9503$ and $L^{2}(0.05)=0.0998$.

The comparison of plans based on noncentral Student's $t$-distribution with those based on the Wallis (1947) approximation reveals low advantages of the former, particularly for lower values of LQL. However, the use of rigorous representations avoids the risk of designing unfeasible plans due to violations of the conditions in the controlled points.

\section{Double Acceptance Sampling Plans}

The design of double sampling plans, for the case of $\sigma$ unknown, is represented as $\mathcal{S}_{2}\left(n_{s 1} \quad k_{r 1} \quad k_{a} ; n_{s 2} \quad k_{r 2}\right)$, with $n_{s 1}, n_{s 2} \in \mathbb{N}$ and $k_{r 1}, k_{a}, k_{r 2} \in \mathbb{R}$, where $n_{s 1}$ is the size of the first sample, $n_{s 2}$ is the size of the second sample, $k_{r 1}$ is the rejection constant to achieve a decision based on the first sample (first stage of decision), $k_{r 2}$ is the rejection constant for deciding after the second sample is taken (second stage of decision), and $k_{a}$ is 
the acceptance constant to accept the lot based on the first sample (first stage of decision). The mechanics is as follows:

1. Sample $n_{s 1}$ items of a lot and determine the quality characteristic of each one, $X_{1}, \ldots, X_{n_{s 1}}$.

2. Determine the average, $\bar{X}_{1}$, and the standard deviation, $s_{1}$, of the sample, by Eq. (4).

3. Determine $t_{1}=\left(U-\bar{X}_{1}\right) / s_{1}$.

4. If $t_{1} \geq k_{a}$, accept the lot.

5. If $t_{1}<k_{r 1}$, reject the lot.

6. If $k_{r 1} \leq t_{1}<k_{a}$, sample $n_{s 2}$ items of the lot and determine the quality characteristic of each one, $X_{n_{s 1}+1}, \ldots, X_{n_{s 1}+n_{s 2}}$.

7. Calculate the average of the second sample and the combined statistics:

$$
\begin{aligned}
& \bar{X}_{2}=\frac{1}{n_{s 2}} \sum_{i=n_{s 1}+1}^{n_{s 1}+n_{s 2}} X_{i} \\
& \bar{X}_{t}=\frac{n_{s 1}}{n_{s 1}+n_{s 2}} \bar{X}_{1}+\frac{n_{s 2}}{n_{s 1}+n_{s 2}} \bar{X}_{2} ; \quad s_{t}=\sqrt{\frac{\sum_{i=1}^{n_{s 1}+n_{s 2}}\left(X_{i}-\bar{X}_{t}\right)^{2}}{n_{s 1}+n_{s 2}-1} .} .
\end{aligned}
$$

8. Determine $t_{2}=\left(U-\bar{X}_{t}\right) / s_{t}$.

9. If $t_{2} \geq k_{r 2}$, accept the lot; otherwise, reject it.

\subsection{Operating Characteristic Curve}

The design of double AS plans for controlling nonconforming proportions has been addressed by several authors by employing the OC curve concept, which requires expressions to compute analytically or numerically the acceptance probability in both decision stages. The general representation of the OC curve is given by

$$
L(p)=P_{a}\left(T_{1} \geq \sqrt{n_{\sigma 1}} k_{a}\right)+P_{a}\left[\left(\sqrt{n_{\sigma 1}} k_{r 1} \leq T_{1}<\sqrt{n_{\sigma 1}} k_{a}\right) \wedge\left(T_{2} \geq \sqrt{n_{\sigma 1}+n_{\sigma 2}} k_{r 2}\right)\right] .
$$

For the sake of simplicity, $P_{a}\left(T_{1} \geq \sqrt{n_{\sigma 1}} k_{a}\right)$ is to be designated $P_{a 1}$, denoting the acceptance probability of the lot based on the first sample, and $P_{a}\left[\left(\sqrt{n_{\sigma 1}} k_{r 1} \leq T_{1}<\right.\right.$ $\left.\left.\sqrt{n_{\sigma 1}} k_{a}\right) \wedge\left(T_{2} \geq \sqrt{n_{\sigma 1}+n_{\sigma 2}} k_{r 2}\right)\right]$ as $P_{a 2}$, representing the acceptance probability based on the second sample. The acceptance probability of the second decision stage is defined in a bivariate region $\Omega=T_{1} \times T_{2}$, with

$$
\Omega=\left\{\left(T_{1}, T_{2}\right) \in \mathbb{R}^{2}:\left(\sqrt{n_{\sigma 1}} k_{r 1} \leq T_{1}<\sqrt{n_{\sigma 1}} k_{a}\right) \wedge\left(T_{2} \geq \sqrt{n_{\sigma 1}+n_{\sigma 2}} k_{r 2}\right)\right\} .
$$

For the case of $\sigma$ known, the OC curve is as follows (Bowker and Goode, 1952):

$$
\begin{aligned}
L^{4}(p)= & \Phi\left[\sqrt{n_{\sigma 1}}\left(\Phi^{-1}(1-p)-k_{a}\right)\right]+\int_{A}^{B} \Phi\left\{\sqrt { \frac { n _ { \sigma 1 } + n _ { \sigma 2 } } { n _ { \sigma 2 } } } \left[\sqrt{n_{\sigma 1}+n_{\sigma 2}} \times\right.\right. \\
& \left.\left.\times\left(\Phi^{-1}(1-p)-k_{r 2}\right)+\sqrt{\frac{n_{\sigma 1}}{n_{\sigma 1}+n_{\sigma 2}}} t\right]\right\} \frac{\mathrm{d} \Phi(t)}{\mathrm{d} t} \mathrm{~d} t,
\end{aligned}
$$




$$
\begin{aligned}
& A=\left[k_{r 1}-\Phi^{-1}(1-p)\right] \sqrt{n_{\sigma 1}}, \\
& B=\left[k_{a}-\Phi^{-1}(1-p)\right] \sqrt{n_{\sigma 1}},
\end{aligned}
$$

with the first term of $L^{4}(p)$ standing for $P_{a 1}$ and the second for $P_{a 2}$.

For processes with unknown $\sigma$, the statistics $\sqrt{n_{s 1}}\left(U-\bar{X}_{1}\right) / s_{1}$ and $\sqrt{n_{s 1}+n_{s 2}}(U-$ $\left.\bar{X}_{t}\right) / s_{t}$ follow noncentral Student's $t$-distributions with different degrees of freedom and means. Furthermore, the distribution of $\sqrt{n_{s 1}+n_{s 2}}\left(U-\bar{X}_{t}\right) / s_{t}$ depends on that of $\sqrt{n_{s 1}}\left(U-\bar{X}_{1}\right) / s_{1}$. The computation of $P_{a 2}$ is very demanding, since it involves the calculation of the cdf for a bivariate noncentral Student's $t$-distribution, here represented as $\Upsilon\left(m_{1}, z_{1} ; m_{2}, z_{2}\right)$, where $m_{1}$ and $m_{2}$ are degrees of freedom in each dimension, and $z_{1}$ and $z_{2}$ are noncentrality parameters. For double AS plans, $m_{1}=n_{s 1}-1, m_{2}=n_{s 1}+n_{s 2}-2$, $z_{1}=\sqrt{n_{s 1}} \Phi^{-1}(1-p)$, and $z_{2}=\sqrt{n_{s 1}+n_{s 2}} \Phi^{-1}(1-p)$.

To lower the computational complexity of the design of double AS plans with $\sigma$ unknown, Sommers (1981) assumed that the statistic $\sqrt{n_{s 1}+n_{s 2}}\left(U-\bar{X}_{t}\right) / s_{t} \sim \mathcal{N}(\mu+$ $\left.k_{r 2} \sigma, \sigma^{2} \times\left(1 /\left(n_{s 1}+n_{s 2}\right)+k_{r 2}^{2} /\left(2 n_{s 1}+2 n_{s 2}-1\right)\right)\right)$. This assumption, based on the approximation proposed by Wallis (1947), relates the total sample size for plans with $\sigma$ known to the corresponding sample size for plans with $\sigma$ unknown and simplifies the determination of acceptance probability $P_{a 2}$ :

$$
n_{\sigma 1}+n_{\sigma 2}=\frac{n_{s 1}+n_{s 2}}{1+k_{r 2}^{2}\left(n_{s 1}+n_{s 2}\right) /\left(2 n_{s 1}+2 n_{s 2}-1\right)} .
$$

The works that use this strategy are not clear regarding the distribution that approximates the statistic $\sqrt{n_{s 1}}\left(U-\bar{X}_{1}\right) / s_{1}$, employed to model the acceptance probability in the first stage of decision. However, if one considers that $\sqrt{n_{s 1}}\left(U-\bar{X}_{1}\right) / s_{1} \sim$ $\mathcal{N}\left(\mu+k_{r 2} \sigma, \sigma^{2} \times\left(1 /\left(n_{s 1}\right)+k_{r 2}^{2} /\left(2 n_{s 1}-1\right)\right)\right)$, similarly to what was assumed in Section 3 , the relations between each of the sample sizes required by plans for $\sigma$ known and $\sigma$ unknown become respectively

$$
\begin{aligned}
& n_{\sigma 1}=\frac{n_{s 1}}{1+k_{r 2}^{2} n_{s 1} /\left(2 n_{s 1}-1\right)}, \\
& n_{\sigma 2}=\frac{n_{s 2}}{1+k_{r 2}^{2} n_{s 2} /\left(2 n_{s 2}-1\right)} .
\end{aligned}
$$

The combination of Eqs. (36-38) with Eqs. (33-35) allows us to the derive the first OC curve to represent double AS plans:

$$
\begin{gathered}
L^{5}(p)=\Phi\left[\sqrt{n_{\sigma 1}}\left(\Phi^{-1}(1-p)-k_{a}\right)\right]+\int_{A}^{B} \Phi\left\{\sqrt { \frac { n _ { \sigma 1 } + n _ { \sigma 2 } } { n _ { \sigma 2 } } } \left[\sqrt{n_{\sigma 1}+n_{\sigma 2}} \times\right.\right. \\
\left.\left.\times\left(\Phi^{-1}(1-p)-k_{r 2}\right)+\sqrt{\frac{n_{\sigma 1}}{n_{\sigma 1}+n_{\sigma 2}}} t\right]\right\} \frac{\mathrm{d} \Phi(t)}{\mathrm{d} t} \mathrm{~d} t \\
A=\left[k_{r 1}-\Phi^{-1}(1-p)\right] \sqrt{n_{\sigma 1}} \\
B=\left[k_{a}-\Phi^{-1}(1-p)\right] \sqrt{n_{\sigma 1}} \\
n_{\sigma 1}=\frac{n_{s 1}}{1+k_{r 2}^{2} n_{s 1} /\left(2 n_{s 1}-1\right)},
\end{gathered}
$$




$$
\begin{aligned}
n_{\sigma 2} & =\frac{n_{s 2}}{1+k_{r 2}^{2} n_{s 2} /\left(2 n_{s 2}-1\right)}, \\
n_{\sigma 1}+n_{\sigma 2} & =\frac{n_{s 1}+n_{s 2}}{1+k_{r 2}^{2}\left(n_{s 1}+n_{s 2}\right) /\left(2 n_{s 1}+2 n_{s 2}-1\right)} .
\end{aligned}
$$

Another alternative addressed in this work consists in assuming that the statistic $\sqrt{n_{s 1}}\left(U-\bar{X}_{1}\right) / s_{1} \sim \mathcal{N}\left(\mu+k_{a} \sigma, \sigma^{2} \times\left(1 /\left(n_{s 1}\right)+k_{a}^{2} /\left(2 n_{s 1}-1\right)\right)\right)$. This assumption leads to the following OC curve:

$$
\begin{aligned}
L^{6}(p)= & \Phi\left[\sqrt{n_{\sigma 1}}\left(\Phi^{-1}(1-p)-k_{a}\right)\right]+\int_{A}^{B} \Phi\left\{\sqrt { \frac { n _ { \sigma 1 } + n _ { \sigma 2 } } { n _ { \sigma 2 } } } \left[\sqrt{n_{\sigma 1}+n_{\sigma 2}} \times\right.\right. \\
& \left.\left.\times\left(\Phi^{-1}(1-p)-k_{r 2}\right)+\sqrt{\frac{n_{\sigma 1}}{n_{\sigma 1}+n_{\sigma 2}}} t\right]\right\} \frac{\mathrm{d} \Phi(t)}{\mathrm{d} t} \mathrm{~d} t, \\
A= & {\left[k_{r 1}-\Phi^{-1}(1-p)\right] \sqrt{n_{\sigma 1}}, } \\
B= & {\left[k_{a}-\Phi^{-1}(1-p)\right] \sqrt{n_{\sigma 1}}, } \\
n_{\sigma 1}= & \frac{n_{s 1}}{1+k_{a}^{2} n_{s 1} /\left(2 n_{s 1}-1\right)}, \\
n_{\sigma 2}= & \frac{n_{s 1}+n_{s 2}}{1+k_{r 2}^{2}\left(n_{s 1}+n_{s 2}\right) /\left(2 n_{s 1}+2 n_{s 2}-1\right)}-\frac{n_{s 1}}{1+k_{a}^{2} n_{s 1} /\left(2 n_{s 1}-1\right)}, \\
n_{\sigma 1}+n_{\sigma 2}= & \frac{n_{s 1}+n_{s 2}}{1+k_{r 2}^{2}\left(n_{s 1}+n_{s 2}\right) /\left(2 n_{s 1}+2 n_{s 2}-1\right)} .
\end{aligned}
$$

To address the problem of explicitly computing the OC curve based on the bivariate noncentral Student's $t$-distribution, Bowker and Goode (1952) derived closed-form formulas, employed for designing plans at given combinations of AQL, LQL, $\alpha$, and $\beta$. The procedure, standing on a rigorous treatment of noncentral Student's $t$-statistics, was not able to cope with general AS plans because the geometry of the region $\Omega$, used to compute $P_{a 2}$, depends on the set of parameters to determine.

Assuming that $\bar{X}_{1}$ and $\bar{X}_{t}$ follow normal distributions and $s_{1}$ and $s_{t}$ are approximated by $\chi_{r}^{2}$-distributions, where $r$ is the number of degrees of freedom, Krumbholz and Rohr (2006) proposed an ontological structure to rigorously determine $P_{a 2}$. For each different class found, corresponding to different features of the region $\Omega$, the authors derived expressions for $P_{a 2}$. The first step of the approach consists in finding the class corresponding to the plan to design. Afterward, the formula derived for such a class is used to compute $P_{a 2}$, and subsequently, the OC curve. This strategy revealed that some of the double AS plans found in the literature are unfeasible if rigorous noncentral Student's $t$-distributions are considered. Indeed, constraint violations were detected at point (LQL, $\beta$ ).

In our work, we employ the strategy proposed by Krumbholz and Rohr (2006) and address the design of AS plans falling into the ontological class with $k_{r 1}>0$, $k_{a} \sqrt{n_{s 1} /\left(n_{s 1}-1\right)} \geq Q$, and $k_{r 1} \sqrt{n_{s 1} /\left(n_{s 1}-1\right)} \geq Q$, with

$$
Q=\sqrt{\frac{n_{s 1}\left(n_{s 1}+n_{s 2}\right) k_{r 2}^{2}-n_{s 2}\left(n_{s 1}+n_{s 2}-1\right)}{\left(n_{s 1}+n_{s 2}\right)\left(n_{s 1}+n_{s 2}-1\right)}} .
$$


The OC curve is therefore computed by

$$
L^{7}(p)=P_{a 1}+P_{a 2}
$$

where $P_{a 1}=\Psi\left[\sqrt{n_{s 1}} k_{a} \mid n_{s 1}-1, \sqrt{n_{s 1}} \Phi^{-1}(1-p)\right]$, and the cdf of the noncentral Student's $t$-distribution is computed through the series expansion methodology presented in Section 2. The term $P_{a 2}$ is handled through the formulas proposed by Krumbholz and Rohr (2006), combined with numerical integration techniques employed to compute the three-dimensional integral term involved (Eq. 53). Considering the relations developed by Krumbholz and Rohr (2006), the acceptance probability associated with the second stage of decision is given by

$$
\begin{aligned}
& P_{a 2}=\int_{0}^{+\infty} \int_{a_{1}\left(w_{1}\right)}^{a_{2}\left(w_{1}\right)} \int_{0}^{K(y)-w_{1}}\left\{\Phi\left[E_{2}\left(w_{1}, y, w_{2}\right)\right]-\Phi\left[E_{1}\left(w_{1}, y, w_{2}\right)\right]\right\} \\
& \times g_{n_{s 2}-1}\left(w_{2}\right) \times \frac{\mathrm{d} \Phi(y)}{\mathrm{d} y} g_{n_{s 1}-1}\left(w_{1}\right) \mathrm{d} w_{2} \mathrm{~d} y \mathrm{~d} w_{1}, \\
& E_{2}\left(w_{1}, y, w_{2}\right)=-D(y)+\sqrt{H\left(w_{1}, y, w_{2}\right)}, \\
& E_{1}\left(w_{1}, y, w_{2}\right)=-D(y)-\sqrt{H\left(w_{1}, y, w_{2}\right)}, \\
& D(y)=\frac{A(y) B+k_{r 2}^{2} y\left(n_{s 1}+n_{s 2}\right) \sqrt{n_{s 1} n_{s 2}}}{B^{2}-n_{s 1}\left(n_{s 1}+n_{s 2}\right) k_{r 2}^{2}}, \\
& H\left(w_{1}, y, w_{2}\right)=\frac{\left(n_{s 1}+n_{s 2}\right) k_{r 2}^{2} C\left(w_{1}, w_{2}\right)+k_{r 2}^{2} n_{s 2}\left(n_{s 1}+n_{s 2}\right) y^{2}-[A(y)]^{2}}{B^{2}-n_{s 1}\left(n_{s 1}+n_{s 2}\right) k_{r 2}^{2}}+[D(y)]^{2}, \\
& A(y)=\sqrt{n_{s 1}+n_{s 2}-1}\left[\sqrt{n_{s 1}} y+\left(n_{s 1}+n_{s 2}\right) \Phi^{-1}(1-p)\right], \\
& B=\sqrt{n_{s 2}\left(n_{s 1}+n_{s 2}-1\right)}, \\
& C\left(w_{1}, w_{2}\right)=\left(n_{s 1}+n_{s 2}\right)\left(w_{1}+w_{2}\right) \text {, } \\
& K(y)=\frac{\left(n_{s 1}+n_{s 2}\right)\left(n_{s 1}+n_{s 2}-1\right)}{n_{s 1}\left(n_{s 1}+n_{s 2}\right) k_{r 2}^{2}-B^{2}}\left[y+\sqrt{n_{s 1}} \Phi^{-1}(1-p)\right]^{2}, \\
& a_{1}\left(w_{1}\right)=k_{r 1} \sqrt{\frac{n_{s 1}}{n_{s 1}-1}} \sqrt{w_{1}}-\sqrt{n_{s 1}} \Phi^{-1}(1-p), \\
& a_{2}\left(w_{1}\right)=k_{a} \sqrt{\frac{n_{s 1}}{n_{s 1}-1}} \sqrt{w_{1}}-\sqrt{n_{s 1}} \Phi^{-1}(1-p),
\end{aligned}
$$

where $g_{r}(z)$ is the value of the $\chi^{2}$-pdf with $r$ degrees of freedom at $z$ :

$$
g_{r}(z)=\frac{1}{2^{r} \Gamma(r / 2)} z^{r / 2-1} \exp (-z / 2)
$$

and

$$
\frac{\mathrm{d} \Phi(y)}{\mathrm{d} y}=\frac{1}{\sqrt{2 \pi}} \exp \left(-y^{2} / 2\right)
$$


The integration variables are $w_{1}, y$, and $w_{2}$, and univariate Gaussian quadrature formulas are employed in each dimension. The upper limit of the outer integral is approximated by a finite large value in order to avoid the finite-domain error, common in the application of numerical rules to infinite-domain integrals (Gelbard and Seinfeld, 1978). This error is due to neglecting a part of the domain that contributes to the solution. Here, we set the upper limit for the domain of $w_{1}$ to $\left\lceil 2 n_{s 1}\right\rceil$, considering that the maximum of a $\chi_{r}^{2}$-pdf occurs at $r$.

\subsection{Problem Formulation}

The strategy supporting the systematic design of double AS plans also stands on the minimization of the ASN, subject to the constraints in the controlled points on the OC curve (Balamurali and Jun, 2007; Duarte and Saraiva, 2010). The ASN for double AS plans with $\sigma$ known is given by the relation:

$$
\mathrm{ASN}_{\sigma}=n_{\sigma 1}+n_{\sigma 2}\left\{\Phi\left[\sqrt{n_{\sigma 1}}\left(k_{a}-\Phi^{-1}(1-p)\right)\right]-\Phi\left[\sqrt{n_{\sigma 1}}\left(k_{r 1}-\Phi^{-1}(1-p)\right)\right]\right\} .
$$

Similar expressions can be derived for plans with $\sigma$ unknown assuming normal distribution approximations. The value of $\mathrm{ASN}_{\sigma}$ depends on the quality level used for its determination, $p$. Sommers (1981) used the point ( $p_{1}$, AQL) for the calculation. Hamaker (1979) demonstrated that $\mathrm{ASN}_{\sigma}$ curves are unimodal with a maximum located at $p_{\max }$. Feldmann and Krumbholz (2002) derived a relation for the quality level corresponding to the maximum of ASN:

$$
p_{\max }=1-\Phi\left(\frac{k_{a}+k_{r 1}}{2}\right)
$$

Based on this result, the authors used the quality level $p_{\max }$ to derive $\mathrm{ASN}_{\sigma}$, thus obtaining

$$
\mathrm{ASN}_{\sigma}=n_{\sigma 1}+n_{\sigma 2}\left[2 \Phi\left(\sqrt{n_{\sigma 1}} \frac{k_{a}-k_{r 1}}{2}\right)-1\right]
$$

They also proved that the quality level $p_{\max }$ leads to minimal values of ASN, and they determined double AS plans to address this criterion. The algorithm proposed was denominated as ASN-minimax, and its application leads to plans with significant advantages over the plans designed by Sommers (1981), since the values of $\mathrm{ASN}_{\sigma}$ are lower for similar quality levels.

Here, the ASN-minimax criterion is also used as objective function. The equivalent ASN for plans with $\sigma$ unknown is denoted as $\mathrm{ASN}_{s}$. For AS plans standing on normal distribution approximations, $n_{\sigma 1}$ and $n_{\sigma 2}$ are functions of $n_{s 1}$ and $n_{s 2}$, leading to problem $\mathcal{P}_{2 a}$ :

$$
\begin{array}{cl}
\min _{n_{s 1}, n_{s 2}, k_{r 1}, k_{a}, k_{r 2}} & n_{\sigma 1}+n_{\sigma 2}\left[2 \Phi\left(\sqrt{n_{\sigma 1}} \frac{k_{a}-k_{r 1}}{2}\right)-1\right] \\
\text { s.t. } & L^{5}\left(p_{1}\right) \geq 1-\alpha \\
& L^{5}\left(p_{2}\right) \leq \beta
\end{array}
$$

Eqs. (40-44)

$$
\Phi^{-1}\left(1-p_{2}\right)<k_{r 1} \leq k_{r 2} \leq k_{a}<\Phi^{-1}\left(1-p_{1}\right), \quad n_{s 1}, n_{s 2}, n_{\sigma 1}, n_{\sigma 2} \in \mathbb{N} .
$$

This problem corresponds to the OC curve derived for $\sqrt{n_{s 1}}\left(U-\bar{X}_{1}\right) / s_{1} \sim \mathcal{N}(\mu+$ $\left.k_{r 2} \sigma, \sigma^{2} \times\left(1 /\left(n_{s 1}\right)+k_{r 2}^{2} /\left(2 n_{s 1}-1\right)\right)\right)$. The problem arising from the assumption that 
$\sqrt{n_{s 1}}\left(U-\bar{X}_{1}\right) / s_{1} \sim \mathcal{N}\left(\mu+k_{a} \sigma, \sigma^{2} \times\left(1 /\left(n_{s 1}\right)+k_{a}^{2} /\left(2 n_{s 1}-1\right)\right)\right)$ and $\sqrt{n_{s 1}+n_{s 2}}(U-$ $\left.\bar{X}_{t}\right) / s_{t} \sim \mathcal{N}\left(\mu+k_{r 2} \sigma, \sigma^{2} \times\left(1 /\left(n_{s 1}\right)+k_{r 2}^{2} /\left(2 n_{s 1}-1\right)\right)\right)$ is designated $\mathcal{P}_{2 b}$ :

$$
\begin{array}{cl}
\min _{n_{s 1}, n_{s 2}, k_{r 1}, k_{a}, k_{r 2}} & n_{\sigma 1}+n_{\sigma 2}\left[2 \Phi\left(\sqrt{n_{\sigma 1}} \frac{k_{a}-k_{r 1}}{2}\right)-1\right] \\
\text { s.t. } & L^{6}\left(p_{1}\right) \geq 1-\alpha \\
& L^{6}\left(p_{2}\right) \leq \beta
\end{array}
$$

Eqs. (46-50)

$$
\Phi^{-1}\left(1-p_{2}\right)<k_{r 1} \leq k_{r 2} \leq k_{a}<\Phi^{-1}\left(1-p_{1}\right), \quad n_{s 1}, n_{s 2}, n_{\sigma 1}, n_{\sigma 2} \in \mathbb{N} \text {. }
$$

The integral terms involved in the OC curves of problems $\mathcal{P}_{2 a}$ and $\mathcal{P}_{2 b}$ are handled with Gaussian quadrature formulas based on eight collocation points corresponding to the zeros of seventh-order Legendre polynomials (Abramowitz and Stegun, 1972).

To address the problem of determining $\mathrm{ASN}_{s}$ when a rigorous noncentral Student's $t$-distribution is employed, Hilbert (2005) proposed

$$
\begin{aligned}
\operatorname{ASN}_{s}= & n_{s 1}+n_{s 2}\left\{\Psi\left[\sqrt{n_{s 1}} k_{a} \mid n_{s 1}-1, \sqrt{n_{s 1}} \Phi^{-1}\left(1-p_{\text {max }}\right)\right]-\right. \\
& \left.-\Psi\left[\sqrt{n_{s 1}} k_{r 1} \mid n_{s 1}-1, \sqrt{n_{s 1}} \Phi^{-1}\left(1-p_{\max }\right)\right]\right\}
\end{aligned}
$$

and developed an approximation for $p_{\max }$ standing on the assumption that noncentral Student's $t$-distribution is well approximated by normal distribution:

$$
p_{\max } \simeq 1-\Phi\left(\sqrt{n_{s 1}} \frac{\sqrt{2 n_{s 1}+k_{r 1}^{2}} k_{a}+\sqrt{2 n_{s 1}+k_{a}^{2}} k_{r 1}}{\sqrt{2 n_{s 1}+k_{r 1}^{2}}+\sqrt{2 n_{s 1}+k_{a}^{2}}}\right) .
$$

We will use Eq. (78) as an equality constraint to relate $p_{\max }$ with the sampling plan parameters. The resulting optimization problem, designated as problem $\mathcal{P}_{2 c}$, can thus be formulated:

$$
\begin{array}{ll}
\min _{n_{s 1}, n_{s 2}, k_{r 1}, k_{a}, k_{r 2}} & n_{s 1}+n_{s 2}\left\{\Psi\left[\sqrt{n_{s 1}} k_{a} \mid n_{s 1}-1, \sqrt{n_{s 1}} \Phi^{-1}\left(1-p_{\max }\right)\right]\right. \\
& \left.-\Psi\left[\sqrt{n_{s 1}} k_{r 1} \mid n_{s 1}-1, \sqrt{n_{s 1}} \Phi^{-1}\left(1-p_{\max }\right)\right]\right\} \\
\text { s.t. } & L^{7}\left(p_{1}\right) \geq 1-\alpha \\
& L^{7}\left(p_{2}\right) \leq \beta \\
& k_{r 1} \sqrt{\frac{n_{s 1}}{n_{s 1}-1}} \geq \sqrt{\frac{n_{s 1}\left(n_{s 1}+n_{s 2}\right) k_{r 2}^{2}-n_{s 2}\left(n_{s 1}+n_{s 2}-1\right)}{\left(n_{s 1}+n_{s 2}\right)\left(n_{s 1}+n_{s 2}-1\right)}}
\end{array}
$$

Eqs. (13-20, 53-65, 78)

$$
\Phi^{-1}\left(1-p_{2}\right)<k_{r 1} \leq k_{r 2} \leq k_{a}<\Phi^{-1}\left(1-p_{1}\right), \quad n_{s 1}, n_{s 2} \geq 1 .
$$

The integration in the domains of $w_{1}$ and $w_{2}$ is carried out with Gaussian quadrature formulas based on 20 collocation points. The density of the grid used aims at guaranteeing numerical accuracy for large domains, which may occur for particular parameter combinations. The integral in the domain of $y$ is also computed through a Gaussian quadrature 
rule based on eight collocation points. The domains of inner integrals are dependent on $w_{1}$, thus requiring the conformation of the numerical rules to the limits, which is done by redistributing the collocation points.

The plans proposed by Sommers (1981) were determined using normal Wallis (1947) approximations. The solution procedure used additional relations between the parameters to reduce the space of feasible solutions to enumerate, with the following assumptions being made: (i) $k_{r 1}=k_{r 2}$ and (ii) $n_{s 1}=n_{s 2}$. AS plans without these constraints can easily be designed, provided that efficient mathematical programming algorithms are employed. Moreover, plans designed without any constraint relating the parameters proved to outperform plans determined considering additional constraints (Duarte and Saraiva, 2010). This result was first observed by Feldmann and Krumbholz (2002), and of late by Duarte and Saraiva (2010). Both works proved that AS plans with unequal sample size and rejection constant (USS\&RC) lead very often to lower values of $\mathrm{ASN}_{s}$. Here, it is assumed that the design of ASN-minimax plans does not involve any additional relation between the parameters.

The AS plans of Sommers (1981), based on equal values of $n_{s 1}$ and $n_{s 2}$, minimize the administrative complexity. However, USS\&RC plans allow to reach similar risks, minimizing the cost of sampling and testing because small sample sizes are required.

Problem $\mathcal{P}_{2 c}$ is much more complex than problems $\mathcal{P}_{2 a}$ and $\mathcal{P}_{2 b}$ due to the numerical requirements needed to compute noncentral Student's $t$-statistics. Its solution, involving a more complex objective function, additional variables, and equality constraints, increases the calculation efforts required. To improve the computational efficiency, we first solve problem $\mathcal{P}_{2 a}$ to produce a feasible initial solution, subsequently provided to problem $\mathcal{P}_{2 c}$, which is then solved also employing GAMS/CONOPT. The solution achieved from $\mathcal{P}_{2 a}$ also allows us to set the number of terms to use in the calculation of the noncentral Student's $t$-cdf (Eq. 14), as well as the upper limit for the integral corresponding to variable $w_{1}$ involved in the computation of $P_{a 2}$.

\subsection{Results}

To demonstrate the robustness and accuracy of our approach, we determined the AS plans for $p_{1}=0.02, \alpha=0.05$, and $\beta=0.1$, with $p_{2}$ varying between 0.03 and 0.2 . The range of cases considered aims mainly to compare the accuracy of our approach, combined with the rigorous treatment of noncentral Student's $t$-cdf, with the plans listed in Sommers (1981), determined assuming normal distribution approximations and enumerative solution schemes. Extensive tables of plans can be produced by changing the producer's and consumer's risks and quality level requirements.

Table 2 compares USS\&RC plans, obtained with the $L^{5}(p)$ OC curve, with the plans of Sommers (1981). Our plans lead to larger values of $\mathrm{ASN}_{s}$ because they use the quality level $p_{\max }$ in the calculation instead of $p_{1}$, used by Sommers (1981). The value of $p_{\max }$ for the AS plan to meet $\mathrm{AQL}=0.02, \mathrm{LQL}=0.05, \alpha=0.05$, and $\beta=0.10$ is 0.0340 . The value of $\mathrm{ASN}_{s}$ based on the sample size of the corresponding plan proposed by Sommers (1981) for this quality level is 146.7 , much larger than ours (117.6). Our approach leads to a consistent trend in the acceptance and rejection constants, a decrease being observed as the LQL increases. Another point to stress is that our strategy does not require any additional constraint between the parameters.

Tables 3 and 4 show the plans designed with $L^{6}(p)$ and $L^{7}(p)$ OC curves, respectively. Both lead to larger values of $\mathrm{ASN}_{s}$ than those required by plans based on $L^{5}(p)$, presented 
Table 2

Double sampling plans for $\alpha=0.05$ and $\beta=0.1$ ( $\sigma$ unknown)

\begin{tabular}{|c|c|c|c|c|c|c|c|c|c|c|c|}
\hline \multirow[b]{2}{*}{$p_{1}$} & \multirow[b]{2}{*}{$p_{2}$} & \multicolumn{6}{|c|}{ USS\&RC plans based on $L^{5}(p)$} & \multicolumn{4}{|c|}{ Sommers (1981) plans } \\
\hline & & $n_{s 1}$ & $n_{s 2}$ & $k_{r 1}$ & $k_{r 2}$ & $k_{a}$ & $\mathrm{ASN}_{s}$ & $n_{s 1}$ & $k_{r 1}$ & $k_{a}$ & $\mathrm{ASN}_{s}$ \\
\hline 0.02 & 0.03 & 527 & 416 & 1.910 & 1.957 & 2.001 & 719.4 & 600 & 1.94 & 1.99 & 675.8 \\
\hline 0.02 & 0.035 & 263 & 207 & 1.853 & 1.918 & 1.981 & 358.5 & 285 & 1.89 & 1.98 & 334.8 \\
\hline 0.02 & 0.04 & 164 & 128 & 1.803 & 1.883 & 1.963 & 223.0 & 179 & 1.85 & 1.95 & 205.8 \\
\hline 0.02 & 0.045 & 115 & 90 & 1.759 & 1.851 & 1.946 & 156.2 & 122 & 1.81 & 1.95 & 144.5 \\
\hline 0.02 & 0.05 & 86 & 68 & 1.716 & 1.822 & 1.935 & 117.6 & 96 & 1.78 & 1.91 & 110.1 \\
\hline 0.02 & 0.06 & 56 & 44 & 1.646 & 1.770 & 1.908 & 76.3 & 62 & 1.72 & 1.87 & 70.4 \\
\hline 0.02 & 0.07 & 41 & 31 & 1.587 & 1.724 & 1.885 & 55.1 & 46 & 1.67 & 1.83 & 51.6 \\
\hline 0.02 & 0.08 & 31 & 25 & 1.527 & 1.682 & 1.870 & 42.5 & 35 & 1.62 & 1.81 & 39.6 \\
\hline 0.02 & 0.09 & 25 & 20 & 1.475 & 1.644 & 1.856 & 34.3 & 27 & 1.57 & 1.82 & 31.5 \\
\hline 0.02 & 0.10 & 21 & 17 & 1.440 & 1.607 & 1.829 & 28.6 & 22 & 1.53 & 1.82 & 26.1 \\
\hline 0.02 & 0.11 & 19 & 12 & 1.405 & 1.576 & 1.815 & 24.4 & 20 & 1.50 & 1.75 & 22.8 \\
\hline 0.02 & 0.12 & 16 & 12 & 1.374 & 1.541 & 1.790 & 21.1 & 17 & 1.47 & 1.72 & 19.2 \\
\hline 0.02 & 0.13 & 14 & 11 & 1.342 & 1.510 & 1.772 & 18.6 & 15 & 1.43 & 1.74 & 17.4 \\
\hline 0.02 & 0.15 & 11 & 9 & 1.266 & 1.455 & 1.759 & 14.9 & 12 & 1.38 & 1.66 & 13.5 \\
\hline 0.02 & 0.17 & 9 & 7 & 1.176 & 1.410 & 1.774 & 12.3 & 10 & 1.31 & 1.69 & 11.7 \\
\hline 0.02 & 0.20 & 7 & 6 & 1.113 & 1.334 & 1.725 & 9.7 & 8 & 1.23 & 1.70 & 9.6 \\
\hline
\end{tabular}

Table 3

USS\&RC double sampling plans based on $L^{6}(p)$ for $\alpha=0.05$ and $\beta=0.1$ ( $\sigma$ unknown)

\begin{tabular}{lcrrcccr}
\hline$p_{1}$ & $p_{2}$ & $n_{s 1}$ & $n_{s 2}$ & $k_{r 1}$ & $k_{r 2}$ & $k_{a}$ & $\mathrm{ASN}_{s}$ \\
\hline 0.02 & 0.030 & 541 & 406 & 1.909 & 1.958 & 2.000 & 727.7 \\
0.02 & 0.035 & 273 & 200 & 1.851 & 1.920 & 1.978 & 364.4 \\
0.02 & 0.040 & 172 & 122 & 1.800 & 1.886 & 1.958 & 227.7 \\
0.02 & 0.045 & 121 & 86 & 1.754 & 1.855 & 1.941 & 160.1 \\
0.02 & 0.050 & 92 & 64 & 1.712 & 1.827 & 1.924 & 121.0 \\
0.02 & 0.060 & 61 & 40 & 1.638 & 1.777 & 1.895 & 79.0 \\
0.02 & 0.070 & 45 & 28 & 1.575 & 1.733 & 1.867 & 57.5 \\
0.02 & 0.080 & 35 & 22 & 1.519 & 1.695 & 1.841 & 44.6 \\
0.02 & 0.090 & 29 & 17 & 1.474 & 1.659 & 1.812 & 36.2 \\
0.02 & 0.100 & 24 & 14 & 1.411 & 1.626 & 1.806 & 30.3 \\
0.02 & 0.110 & 21 & 12 & 1.388 & 1.597 & 1.766 & 25.9 \\
0.02 & 0.120 & 18 & 11 & 1.339 & 1.569 & 1.755 & 22.6 \\
0.02 & 0.130 & 16 & 9 & 1.283 & 1.541 & 1.753 & 20.0 \\
0.02 & 0.150 & 13 & 7 & 1.203 & 1.492 & 1.728 & 16.2 \\
0.02 & 0.170 & 11 & 6 & 1.166 & 1.448 & 1.671 & 13.5 \\
0.02 & 0.200 & 9 & 5 & 1.137 & 1.389 & 1.576 & 10.7 \\
\hline
\end{tabular}


Table 4

USS\&RC double sampling plans based on $L^{7}(p)$ for $\alpha=0.05$ and $\beta=0.1$ ( $\sigma$ unknown)

\begin{tabular}{lcrrcccr}
\hline$p_{1}$ & $p_{2}$ & $n_{s 1}$ & $n_{s 2}$ & $k_{r 1}$ & $k_{r 2}$ & $k_{a}$ & $\mathrm{ASN}_{s}$ \\
\hline 0.02 & 0.030 & 532 & 408 & 1.912 & 1.958 & 2.003 & 718.6 \\
0.02 & 0.035 & 275 & 198 & 1.862 & 1.920 & 1.978 & 360.0 \\
0.02 & 0.040 & 166 & 129 & 1.810 & 1.887 & 1.966 & 224.2 \\
0.02 & 0.045 & 115 & 93 & 1.764 & 1.857 & 1.956 & 157.3 \\
0.02 & 0.050 & 85 & 72 & 1.722 & 1.829 & 1.949 & 118.8 \\
0.02 & 0.060 & 57 & 47 & 1.660 & 1.781 & 1.923 & 77.3 \\
0.02 & 0.070 & 42 & 33 & 1.602 & 1.738 & 1.907 & 56.2 \\
0.02 & 0.080 & 33 & 25 & 1.551 & 1.701 & 1.896 & 43.6 \\
0.02 & 0.090 & 27 & 21 & 1.505 & 1.666 & 1.888 & 35.3 \\
0.02 & 0.100 & 22 & 17 & 1.465 & 1.635 & 1.876 & 29.6 \\
0.02 & 0.110 & 19 & 15 & 1.428 & 1.606 & 1.868 & 25.3 \\
0.02 & 0.120 & 17 & 13 & 1.393 & 1.579 & 1.863 & 22.1 \\
0.02 & 0.130 & 15 & 11 & 1.363 & 1.554 & 1.855 & 19.6 \\
0.02 & 0.150 & 12 & 9 & 1.307 & 1.507 & 1.846 & 15.8 \\
0.02 & 0.170 & 11 & 8 & 1.256 & 1.465 & 1.841 & 13.3 \\
0.02 & 0.200 & 9 & 6 & 1.191 & 1.408 & 1.833 & 10.6 \\
\hline
\end{tabular}

in Table 2. The values of $\mathrm{ASN}_{s}$ determined with the OC curve $L^{7}(p)$ fall within the range of the other two, both based on normal distribution approximations.

Figure 6 compares the $\mathrm{OC}$ curves of all plans determined for $\mathrm{AQL}=0.02, \mathrm{LQL}=0.05$, $\alpha=0.05$, and $\beta=0.10$, which are quite similar. $L^{7}(p)$ leads to lower values of acceptance probability for higher nonconforming proportion values and overestimates the acceptance probability in the range of low values of $p$. A deeper analysis of the performance of the plans at the controlled points reveals that those resulting from $L^{5}(p)$ and $L^{6}(p)$ violate the constraint at (LQL, $\beta$ ), an observation already made by Krumbholz and Rohr (2006). From a practical point of view, the impact of this violation is often disregarded, since the difference is small. From a theoretical point of view, it deserves some attention because the plan based on $L^{7}(p)$ is the only one that systematically allows to meet the risks considered. Table 5 clarifies this finding and reveals that only the OC curve based on rigorous noncentral Student's $t$-distribution produces feasible plans for this case.

Table 5

Performance of AS plans at the OC controlled points

\begin{tabular}{|c|c|c|c|c|c|c|}
\hline \multirow{2}{*}{$\frac{\text { OC curve }}{\text { Based on Sommers (1981) }}$} & \multicolumn{4}{|c|}{ Plan design } & \multirow{2}{*}{$\frac{L\left(p_{1}\right)}{0.9533}$} & \multirow{2}{*}{$\frac{L\left(p_{2}\right)}{0.1044^{*}}$} \\
\hline & $\mathcal{S}_{2}(96$ & 1.78 & $1.91 ; 96 \quad 1$ & 1.78) & & \\
\hline$L^{5}(p)$ & $\mathcal{S}_{2}(86$ & 1.716 & $1.935 ; 68$ & $1.822)$ & 0.9553 & $0.1089^{*}$ \\
\hline$L^{6}(p)$ & $\mathcal{S}_{2}(92$ & 1.712 & $1.924 ; 64$ & $1.827)$ & 0.9586 & $0.1040^{*}$ \\
\hline$L^{7}(p)$ & $\mathcal{S}_{2}(85$ & 1.722 & $1.949 ; 72$ & $1.829)$ & 0.9503 & 0.0981 \\
\hline
\end{tabular}

*Constraint violation. 


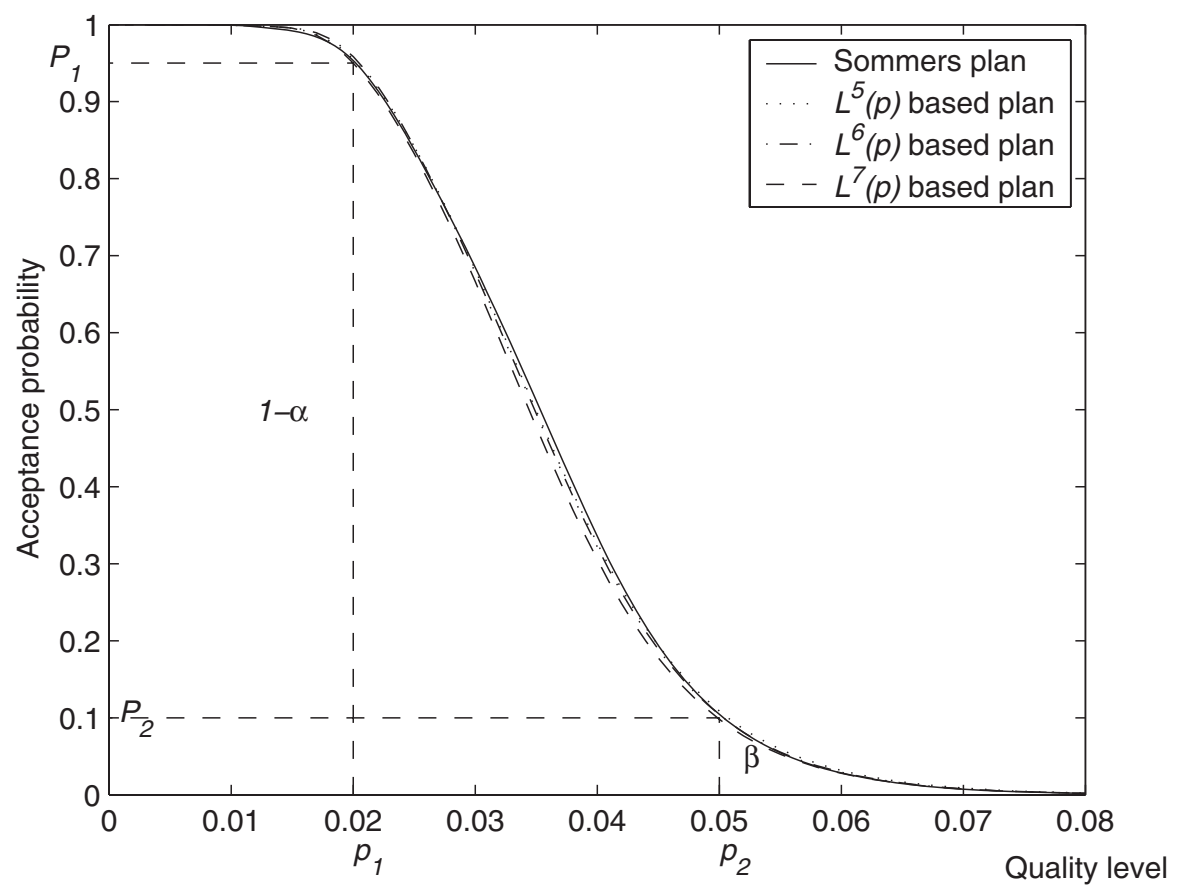

Figure 6. Comparison of OC curves for double AS plans based on rigorous noncentral Student's $t$-distribution for $\mathrm{AQL}=0.02, \mathrm{LQL}=0.05, \alpha=0.05$, and $\beta=0.10$.
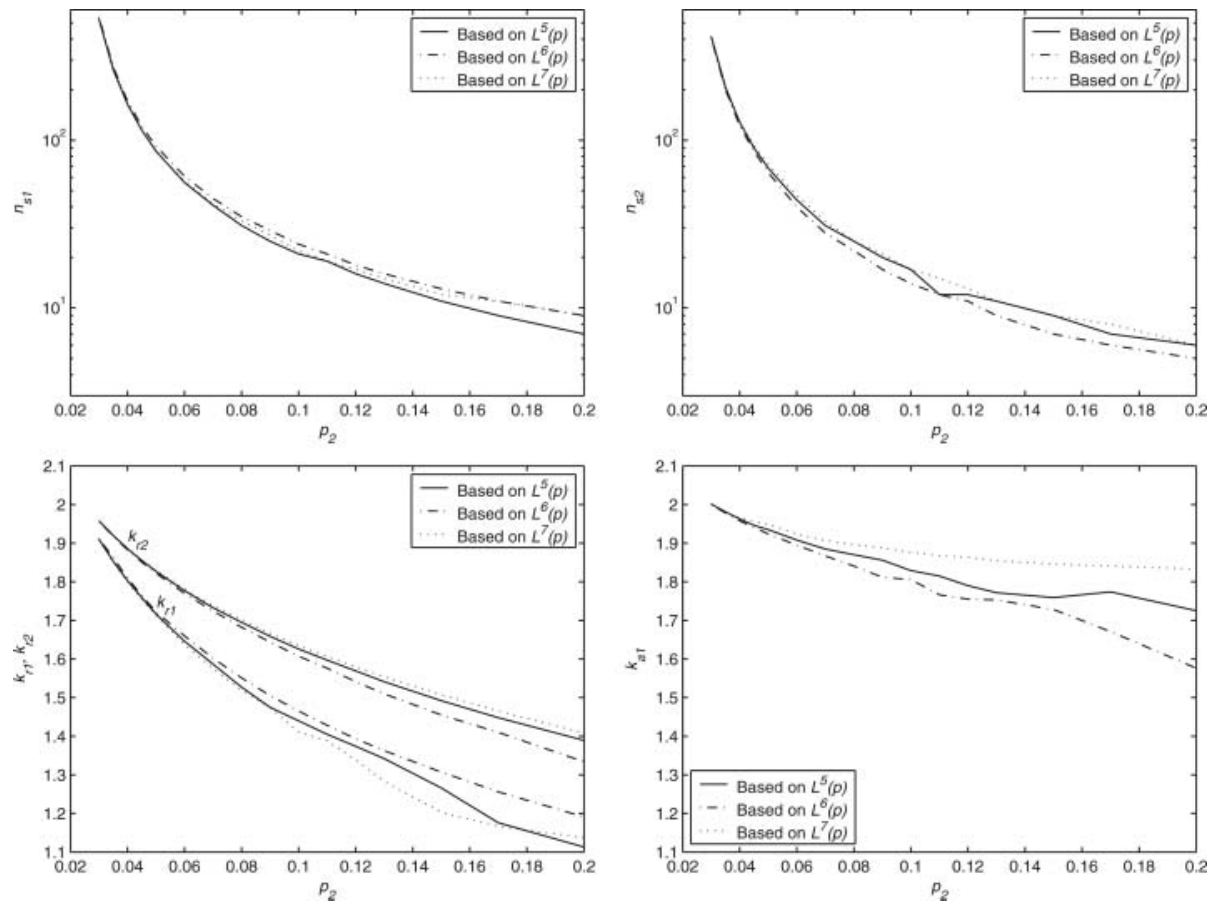

Figure 7. Sensitivity analysis of double AS plans designed for $\mathrm{AQL}=0.02, \alpha=0.05$, and $\beta=0.10$ to $\mathrm{LQL}$. 


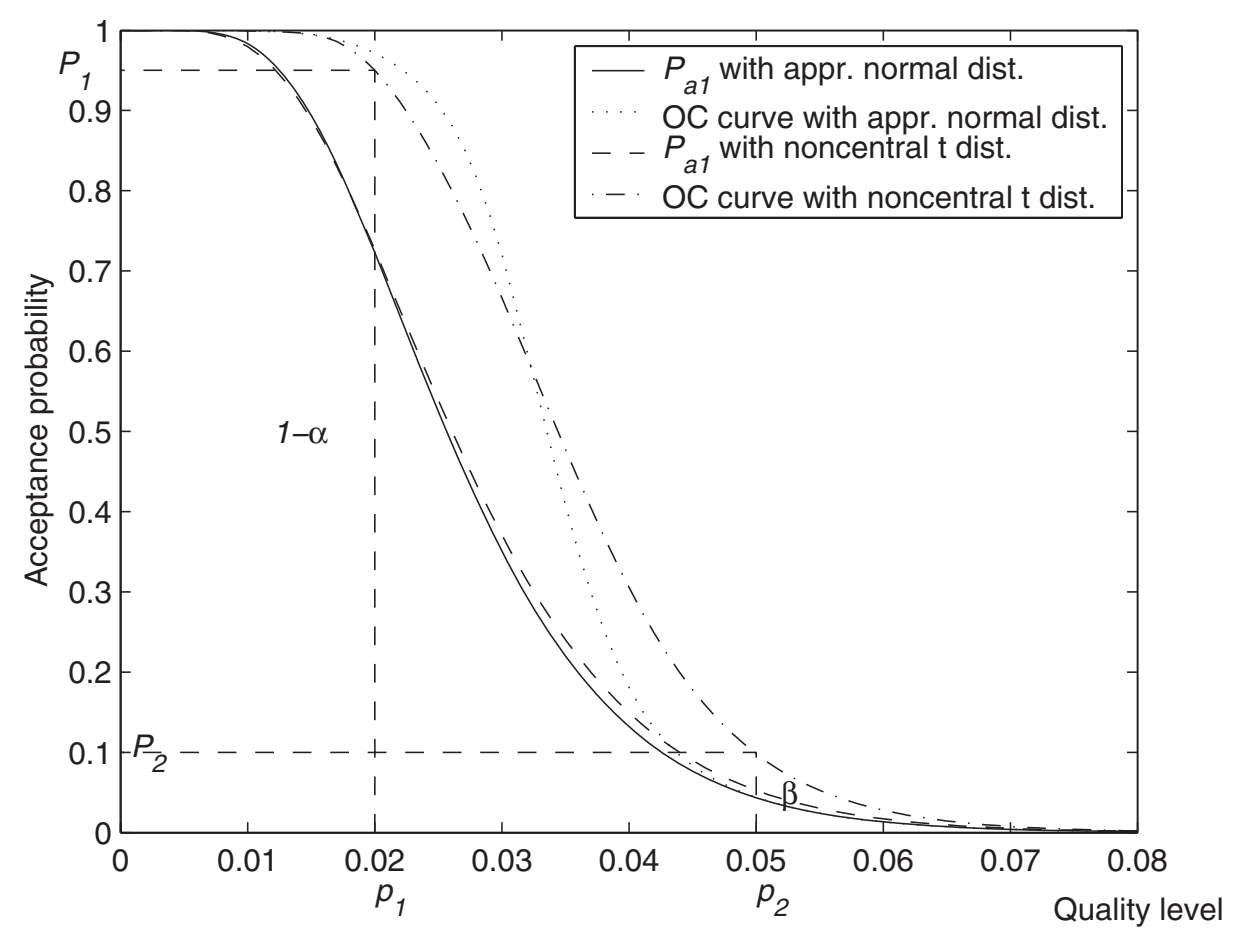

Figure 8. OC curves computed with normal approximation and rigorous noncentral Student's $t$ distribution for plan $\mathcal{S}_{2}(85 \quad 1.722 \quad 1.949 ; 72 \quad 1.829)$.

The sensitivity analysis of the plans produced with the OC curves $L^{5}(p), L^{6}(p)$, and $L^{7}(p)$ reveals that they are very similar for low values of LQL (see Figure 7). This observation agrees with the fact that normal distributions are better approximations of noncentral Student's $t$-statistics for large sample sizes, required by plans designed to meet lower values of LQL. Furthermore, the plans obtained with $L^{7}(p)$ require smaller samples to reach the first stage of decision than those obtained with $L^{6}(p)$. However, this advantage is obtained at the expense of larger samples needed in the second decision stage. Another aspect is that both the difference between the rejection constants $k_{r 2}$ and $k_{r 1}$ and the acceptance constant are higher for $L^{7}(p)$.

Figure 8 illustrates the differences between the OC curves obtained with the normal approximation proposed by Wallis (1947) and that calculated with noncentral Student's $t$ distribution for plan $\mathcal{S}_{2}(85 \quad 1.722 \quad 1.949 ; 72 \quad 1.829)$ obtained for $\mathrm{AQL}=0.02, \mathrm{LQL}=$ $0.05, \alpha=0.05$, and $\beta=0.10$. The components of the OC curves describing the acceptance probability of the first stage of decision are very similar. On the contrary, normal approximation involving both terms $P_{a 1}$ and $P_{a 2}$ overestimates the acceptance probability at AQL and LQL. This trend agrees with the finding that plans based on normal approximations are unfeasible due to the violation of the constraint at the point (LQL, $\beta$ ).

The results shown in Tables 2-4 demonstrate the adequacy of the approach used in dealing with a vast range of cases employing normal approximations or rigorous double $t$ test-based OC curves. It allows a systematic treatment of the AS plan design, demonstrated by the battery of examples handled, with the results being in good agreement with others available in the literature. However, the quality levels required by the producer and the 
Table 6

USS\&RC double sampling plans based on $L^{7}(p)$ for $\alpha=0.05$ and $\beta=0.1$ ( $\sigma$ unknown)

\begin{tabular}{lcrrcccr}
\hline$p_{1}$ & $p_{2}$ & $n_{s 1}$ & $n_{s 2}$ & $k_{r 1}$ & $k_{r 2}$ & $k_{a}$ & $\mathrm{ASN}_{s}$ \\
\hline 0.0010 & 0.0030 & 240 & 189 & 2.806 & 2.898 & 2.988 & 327.4 \\
0.0010 & 0.0035 & 179 & 141 & 2.765 & 2.868 & 2.973 & 244.1 \\
0.0010 & 0.0040 & 142 & 112 & 2.728 & 2.843 & 2.961 & 194.0 \\
0.0010 & 0.0045 & 118 & 93 & 2.697 & 2.820 & 2.949 & 160.8 \\
0.0010 & 0.0050 & 101 & 79 & 2.668 & 2.799 & 2.939 & 137.4 \\
0.0010 & 0.0060 & 78 & 62 & 2.617 & 2.762 & 2.922 & 106.5 \\
0.0010 & 0.0070 & 64 & 51 & 2.575 & 2.730 & 2.905 & 87.3 \\
0.0010 & 0.0080 & 55 & 42 & 2.539 & 2.701 & 2.891 & 74.1 \\
0.0010 & 0.0090 & 48 & 37 & 2.508 & 2.675 & 2.876 & 64.6 \\
0.0010 & 0.0100 & 42 & 33 & 2.468 & 2.653 & 2.876 & 57.4 \\
0.0010 & 0.0110 & 38 & 30 & 2.445 & 2.631 & 2.861 & 51.7 \\
0.0010 & 0.0120 & 35 & 27 & 2.425 & 2.611 & 2.848 & 47.1 \\
0.0010 & 0.0130 & 32 & 25 & 2.398 & 2.593 & 2.843 & 43.3 \\
0.0010 & 0.0150 & 28 & 21 & 2.358 & 2.559 & 2.827 & 37.5 \\
0.0010 & 0.0170 & 25 & 19 & 2.335 & 2.526 & 2.799 & 33.1 \\
0.0010 & 0.0200 & 21 & 16 & 2.263 & 2.489 & 2.806 & 28.4 \\
\hline
\end{tabular}

consumer are in most of the cases, nowadays, remarkably lower than those considered for comparison purposes.

To test our algorithm in more challenging environments, we considered $\mathrm{AQL}=0.001$ and LQL in the range [0.003-0.02]. One aspect to account for in dealing with these scenarios

Table 7

USS\&RC double sampling plans based on $L^{7}(p)$ for $\alpha=0.05$ and $\beta=0.01$ ( $\sigma$ unknown)

\begin{tabular}{lcrrcccr}
\hline$p_{1}$ & $p_{2}$ & $n_{s 1}$ & $n_{s 2}$ & $k_{r 1}$ & $k_{r 2}$ & $k_{a}$ & $\mathrm{ASN}_{s}$ \\
\hline 0.0010 & 0.0030 & 476 & 319 & 2.890 & 2.944 & 3.023 & 625.9 \\
0.0010 & 0.0035 & 356 & 240 & 2.861 & 2.922 & 3.013 & 468.6 \\
0.0010 & 0.0040 & 284 & 192 & 2.835 & 2.902 & 3.004 & 373.7 \\
0.0010 & 0.0045 & 236 & 160 & 2.812 & 2.885 & 2.997 & 310.8 \\
0.0010 & 0.0050 & 202 & 137 & 2.791 & 2.869 & 2.991 & 266.2 \\
0.0010 & 0.0060 & 158 & 107 & 2.757 & 2.841 & 2.977 & 207.6 \\
0.0010 & 0.0070 & 130 & 88 & 2.725 & 2.817 & 2.967 & 170.8 \\
0.0010 & 0.0080 & 111 & 75 & 2.699 & 2.795 & 2.958 & 145.7 \\
0.0010 & 0.0090 & 97 & 66 & 2.675 & 2.776 & 2.950 & 127.4 \\
0.0010 & 0.0100 & 87 & 59 & 2.657 & 2.758 & 2.938 & 113.6 \\
0.0010 & 0.0110 & 78 & 54 & 2.635 & 2.742 & 2.935 & 102.6 \\
0.0010 & 0.0120 & 72 & 49 & 2.621 & 2.726 & 2.924 & 93.8 \\
0.0010 & 0.0130 & 66 & 45 & 2.600 & 2.713 & 2.924 & 86.6 \\
0.0010 & 0.0150 & 57 & 40 & 2.569 & 2.687 & 2.914 & 75.2 \\
0.0010 & 0.0170 & 51 & 35 & 2.544 & 2.664 & 2.903 & 66.8 \\
0.0010 & 0.0200 & 44 & 31 & 2.516 & 2.630 & 2.884 & 57.5 \\
\hline
\end{tabular}


is that normal approximations may not work for extreme tail probabilities (Wallis, 1947). Furthermore, the solution would require OC curves with higher discriminating power (steepness). The results shown in Table 6 are in good agreement with those of Schilling (1982) and demonstrate that our tool is successful in dealing with processes in this range of quality requirements. Schilling and Johnson (1980) recommend employing very small values of $\beta$ for safety purposes. These scenarios are also increasingly demanding for AS plans design tools because they also require highly discriminating OC curves. To test our algorithm for lower values of errors of type II, we consider $\beta=0.01$ and the same range of LQL and AQL values used in Table 6. The results shown in Table 7 extend the ability of the approach to cases with low values of consumer's risk corresponding to inspection for safety purposes.

\section{Conclusion}

This article employs an optimization-based approach for designing AS plans by variables for controlling nonconforming proportions when $\sigma$ is unknown. The approach is applied to single and double AS plans, and uses rigorous algorithmic strategies to compute noncentral Student's $t$-statistics. In the design of single AS plans, noncentral Student's $t$-statistic is employed to determine the acceptance probability of the decision regarding the lot acceptance/rejection. Noncentral Student's $t$-cdf is calculated through a series expansion. A similar approach is used to compute the OC curve representing the acceptance probability of the first stage of decision for double AS plans. The acceptance probability of the second stage of decision in double AS plans required by the OC curve proposed by Krumbholz and Rohr (2006) is determined with Gaussian quadrature rules. AS plans based on rigorous noncentral Student's $t$-statistic are compared with plans derived assuming nonstandard normal approximations and with other plans available in the literature.

Our mathematical programming problem formulation stands on the minimization of the ASN, subject to constraints in the OC curve controlled points, and enables to robustly and accurately cope with the design of single and double AS plans. The relaxed formulation of the optimization problems is solved by employing NLP solvers. This procedure allows one to systematically address any kind of AS plan, guaranteeing that globally optimal solutions are obtained. Furthermore, the feasibility of the AS plans is assured by the solver.

The use of rigorous noncentral Student's $t$-distribution calculation schemes revealed that some of the plans proposed by Sommers (1981) are unfeasible, denoting consistent violations of the constraint holding at LQL. AS plans based on normal distribution approximations were addressed, and two different assumptions were considered: (i) the statistics tested in both stages of decision follow the same normal distribution, and (ii) the statistics tested in each stage of decision follow different normal distribution approximations. The designs obtained with the rigorous treatment of noncentral Student's $t$-statistics are consistent with those produced employing normal distribution approximations. The approach was successfully tested using a battery of scenarios available in the literature, outperforming the results in some of the cases. To analyze its ability to cope with more challenging problems due to the requirement of higher discriminating power, it was also tested in environments characterized by lower AQL and LQL values and different consumer's risks, thus denoting large accuracy.

\section{References}

Abramowitz, M., Stegun, I. (1972). Handbook of Mathematical Functions. New York: Dover Pulications. 
Balamurali, S., Jun, C.-H. (2007). Multiple dependent state sampling plans for lot acceptance based on measurement data. European Journal Operational Research 180:1221-1230.

Bowker, A. H., Goode, H. P. (1952). Sampling Inspection by Variables. New York: McGraw-Hill.

Drud, A. (1985). CONOPT: A GRG code for large sparse dynamic nonlinear optimization problems. Mathematical Programming 31:153-191.

Duarte, B., Saraiva, P. (2010). An optimization-based framework for designing acceptance sampling plans by variables for nonconforming proportions. International Journal Quality and Reliability Engineering 27:794-814.

Duncan, A. (1974). Quality Control and Industrial Statistics. 4th ed. Homewood, IL: Richard D. Irwin.

Evans, J. R. (2005). Total Quality - Management, Organizations and Strategy. 4th ed. Toronto, Canada: Thomson.

Feldmann, B., Krumbholz, W. (2002). ASN-Minimax double sampling plans for variables. Statistical Papers 43:361-377.

Gelbard, F., Seinfeld, J. (1978). Numerical solution of the dynamic equation for particulate systems. Journal of Computational Physics 28:357-375.

Guenther, W. (1978). Evaluation of the probabilities for the noncentral distributions and the difference of two $t$-variables with a desk calculator. Journal of Statistical Computation and Simulation 6:199-206.

Hamaker, H. C. (1979). Acceptance sampling for percent defective by variables and by attributes. Journal of Quality Technology 11:139-148.

Hilbert, M. (2005). Zweifache ASN-Minimax-Variablenprüfpläne für normalverteiltes Merkmal bei unbekannten Parametern. Ph.D. thesis. Hamburg: Universität der Bundeswehr Hamburg.

Krumbholz, W., Rohr, A. (2006). The operating characteristic of double sampling plans by variables when the standard deviation is unknown. Allgemeines Statistisches Archiv 90:233-251.

Lenth, R. (1989). Algorithm AS 243: cumulative distribution function of the non-central $t$ distribution. Applied Statistics 38:185-189.

Mitra, A. (1998). Fundamentals of Quality Control and Improvement. 2nd ed. Upper Saddle River, NJ: Prentice-Hall.

Schilling, E. G. (1982). Acceptance Sampling in Quality Control. New York: Marcel Dekker.

Schilling, E. G., Johnson, L. (1980). Tables for the construction of matched single, double, and multiple sampling plans with application to MIL-STD-105D. Journal of Quality Technology 12:220-229.

Seidel, W. (1997). Is sampling by variables worse than sampling by attributes? A decision theoretic analysis and a new mixed strategy for inspecting individual lots. Sankhya B 59:6-107.

Sommers, D. J. (1981). Two-point double variables sampling plans. Journal of Quality Technology 13:5-30.

Wallis, W. A. (1947). Techniques of Statistical Analysis. New York: McGraw-Hill. 